\title{
Envelope-Aware Flight Management for Loss of Control Prevention Given Rudder Jam
}

\author{
Pedro F. A. Di Donato* \\ National Civil Aviation Agency-Brazil (ANAC), São José dos Campos, SP 12242, Brazil \\ and \\ Sweewarman Balachandran, \pm Kevin McDonough, \\ University of Michigan, Ann Arbor, Michigan 48109 \\ DOI: $10.2514 / 1 . G 000252$
}

\begin{abstract}
The Envelope-Aware Flight Management System is an augmentation to a conventional flight management system that prevents or recovers from loss of control. Loss of control is defined in this work as an excursion outside the admissible flight envelope. The Envelope-Aware Flight Management System combines flight safety assessment and management, envelope estimation, flight planning, system identification, and adaptive control functions. This paper describes integration of envelope estimation, flight planning, and flight safety assessment and management to identify situations of high loss-of-control risk, override the nominal crew/flight-management-system just in time to prevent loss of control, and safely recover the aircraft. The Envelope-Aware Flight Management System is applied to prevent inflight loss of control for a Twin Otter experiencing a rudder jam, a failure that can be identified and modeled a priori. Recovery translates to identifying and maintaining states within the contracted flight envelope, as well as building and following an emergency landing plan that respects the new envelope.
\end{abstract}

$\begin{array}{lll} & \text { Nomenclature } \\ \bar{d} & =\text { flight plan deviation state } \\ F_{1}, F_{2}, F_{3}, F_{4} & =\text { MDP state features } \\ f_{M} & =\text { mode selector switch } \\ M & =\text { mode } \\ n_{\text {turns }} & =\text { number of complete 360-deg turns } \\ O_{\infty} & =\text { in path segment } \\ R_{\infty} & =\text { safe set } \\ p, q, r & =\text { angular rates, deg } / \mathrm{s} \\ \bar{p} & =\text { dynamic pitch state } \\ R & =\text { path planning turn radius, } \mathrm{m} \\ \bar{r} & =\text { MDP dynamic roll } \\ (\mathcal{S}, \mathcal{A}, \mathcal{T}, \mathcal{R}) & =\text { MDP states, actions, transition } \\ u, v, w & =\text { probabilities, and rewards } \\ \bar{v} & =\text { MDP discrete airspeed state } \\ x, y, z & =\text { three-dimensional position, } \mathrm{m} \\ \gamma & =\text { flight path angle, deg } \\ \delta_{e}, \delta_{a}, \delta_{r} & =\text { elevator, aileron, and rudder, deg } \\ \delta_{p} & =\text { power control input, percentage } \\ \eta & =\text { of maximum power } \\ \theta & =\text { distance between waypoints } 3 \text { and } 4, \mathrm{~m} \\ \sigma & =\text { pitch angles, deg } \\ \phi & =\text { reading angle, deg } \\ \psi & = & \text { yaw angle, deg } \\ & & \end{array}$

Received 6 November 2015; revision received 25 March 2016; accepted for publication 2 April 2016; published online 9 August 2016. Copyright $@ 2016$ by Pedro F. A. Di Donato. Published by the American Institute of Aeronautics and Astronautics, Inc., with permission. Copies of this paper may be made for personal and internal use, on condition that the copier pay the per-copy fee to the Copyright Clearance Center (CCC). All requests for copying and permission to reprint should be submitted to CCC at www.copyright.com; employ the ISSN 0731-5090 (print) or 1533-3884 (online) to initiate your request.

*Regulation Specialist (on leave); also Ph.D. Candidate, University of Michigan, Ann Arbor, MI 48109; pdonato@umich.edu.

${ }^{\dagger}$ Graduate Research Assistant, Department of Aerospace Engineering; swee@umich.edu.

${ }^{*}$ Graduate Research Assistant, Department of Aerospace Engineering; kevinmcd@umich.edu.

${ }^{\S}$ Associate Professor, Department of Aerospace Engineering; ematkins@ umich.edu.

ๆProfessor, Department of Aerospace Engineering; ilya@umich.edu.

\section{Introduction}

A VIATION is an efficient and safe means of transport. Flyby-wire technology allows onboard automation to monitor crew inputs and the suite of connected subsystems. Flight control computers reliably follow flight plans by precisely guiding, navigating, and controlling the aircraft to follow trimmed flight states or instrument flight plans. Triply redundant architectures provide statistical guarantees that safety-critical avionics, power, and mechanical systems will function reliably. Despite the high levels of reliability and functionality in modern aircraft, loss of control (LOC) accidents still occur. For this paper, LOC is defined as a condition in which an unusual attitude, attitude rate of change, or aerodynamic state violate normal operating constraints, causing deviation outside the normal flight envelope [1]. Most LOC accidents result from some combination of inappropriate pilot inputs, bad weather, and onboard system failures [1].

Envelope protection systems are available today, preventing specific constraint violations such as minimum airspeed and maximum bank angle [2]. Thresholds that trigger envelope protections are static and thus do not adapt to system or environment changes. Instead, envelope protection disengages when exceptions occur. For certification, adopting conservative envelope protection thresholds facilitates airworthiness compliance. It is simpler to inhibit the envelope protection system than to design a system that would adapt to the adverse condition that could be proven safe for all different scenarios. The crew must then make complex recovery decisions in a limited time, high-stress, high-workload environment. The likelihood of inappropriate crew inputs increases as stress and workload increase and situational awareness degrades. Additionally, current flight envelope protection features do not prevent the crew from deliberately or unintentionally endangering safety of flight (e.g., flying into terrain or a building).

This paper presents the Envelope-Aware Flight Management System (EA-FMS) as an efficient augmentation to a conventional flight management system (FMS) to improve response to potential LOC scenarios by effectively processing critical LOC-related data and generating recovery solutions. EA-FMS combines flight safety assessment and management (FSAM) [3-7] , envelope estimation $[\underline{8}, 9]$, adaptive flight planner [10], and system identification [11] , as illustrated in Fig. 1. For LOC scenarios not modeled offline, adaptive control [12] might also be required. The EA-FMS predicts and prevents or recovers from LOC situations, including stall and unusual attitude, whether induced by environment, crew, onboard failure, or multiple factors. Resiliency can be achieved in cases that can be 


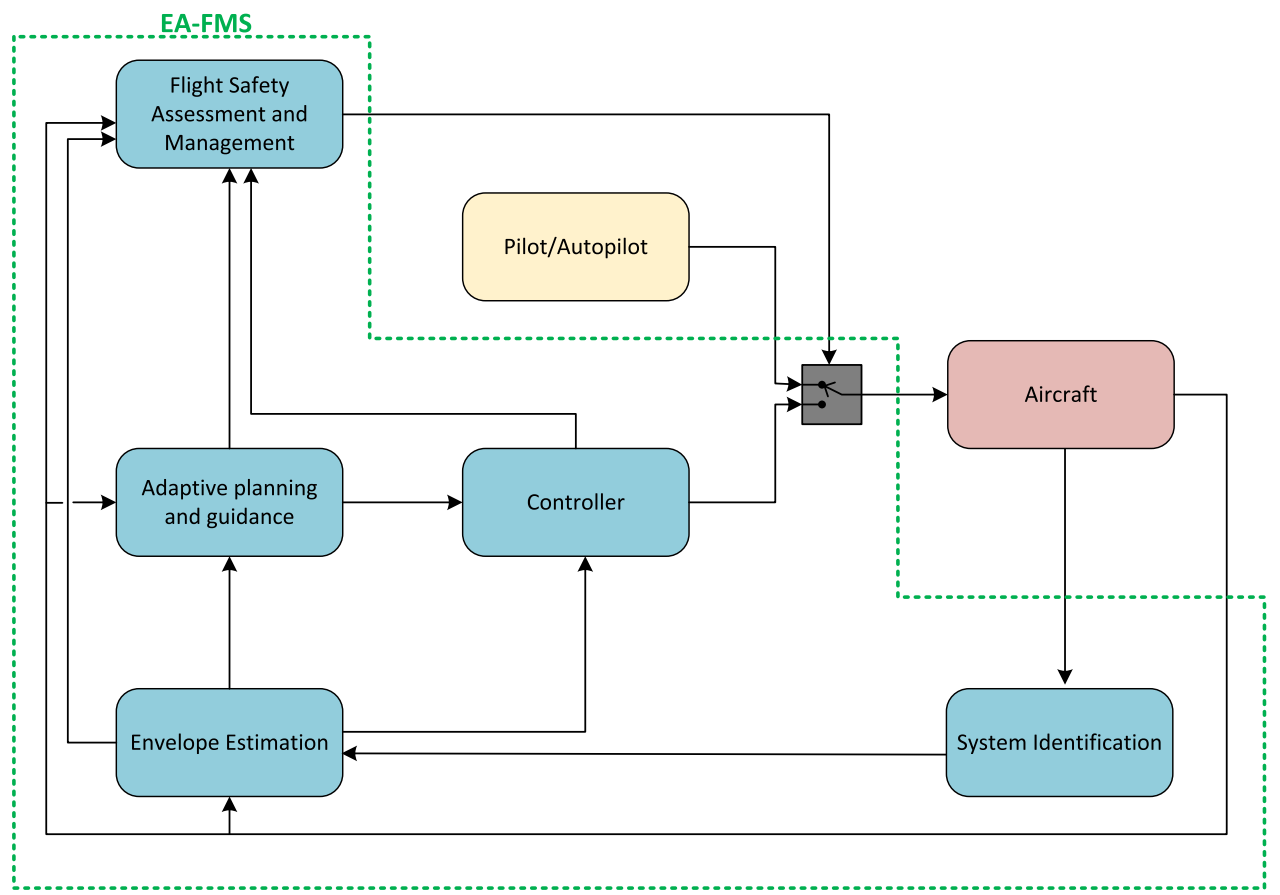

Fig. 1 The envelope-aware flight management system.

anticipated and modeled a priori using an envelope or flight dynamics model database. Envelope estimation and system identification then process incoming data to generate a match with a database exception entry. If online adaptation is required for scenarios that cannot be accurately anticipated, like airframe icing, then envelope estimation and model parameter updates allow LOC risk assessment and handling with respect to new envelope constraints. The EA-FMS flight planner is inactive during nominal flight, engaged only to assist with online envelope discovery [13] or to guide the aircraft to a safe state or a safe emergency landing [14]. Flight safety assessment and management (FSAM) ensures the appropriate control authority is selected in time to prevent or recover from LOC.

The primary contribution of this work is formal integration of the different EA-FMS modules that were separately developed in previous papers. EA-FMS functions and applications are illustrated using an actuator-failure LOC scenario, specifically a rudder jam. This failure causes a nonzero yawing moment and adverse rolling moment due to the airframe's inertial and aerodynamic cross coupling. Jammed rudder failure cases can be accurately characterized offline with compiled model data then rapidly accessed online should this rare but dangerous failure occur.

This paper also contributes a procedure to generate an admissible transition database based on recoverable sets and applies this database to an adaptive flight planner that in turn generates constraintadmissible flight trajectories. This procedure can be applied offline, as in this work, or it could be extended to update flight envelope constraints online, given unmodeled or continuously degrading performance for situations such as structural damage or icing.

A final contribution of this paper is in the application of EA-FMS to prevent loss of control situations related to rudder-jam scenarios. Offline analysis enables database models to be fully validated and verified for the rudder-jam case before flight and supports real-time EA-FMS response in flight. Because the specific failure is characterized in advance, system identification is not used and envelope estimation can be performed offline.

This paper is organized as follows. Section II provides background on related research. Section III formulates envelope estimation, Sec. IV presents path planning, and Sec. $\underline{V}$ describes flight safety assessment and management (FSAM). Section VI summarizes the sequence of offline and online computations required to realize EA-FMS, whereas Sec. VII presents results of the integrated system applied to the rudder-jam case study. Section VIII concludes the paper.

\section{Related Work}

Several methods to identify and mitigate LOC risk have been investigated in previous work. Most have focused on providing cues to the pilot or crew. The Safety Augmentation System (SafAS) [15] is an automation aid to prevent entry into hazardous conditions, such as unfavorable weather. It identifies flight plan deviations and issues warning messages and haptic feedback on hazard detection. Icing Contamination Envelope Protection (ICEPro) [16] identifies airplane performance degradations resulting from ice contamination with online system identification and provides associated cues to pilots. Belcastro and Jacobson [1] proposed the Aircraft Integrated Resilient Safety Assurance and Failsafe Enhancement (AIRSAFE) conceptual architecture for LOC avoidance. AIRSAFE includes online modeling, safety assessment, and resilient control in situations with appreciable LOC risk. Hovakimyan and coworkers [17-19] introduced the Integrated Reconfigurable Control for Vehicle Resilience (iReCoVeR) architecture that combines flight envelope protection, LOC prediction and prevention, fault detection and isolation, and a robust adaptive flight controller to recover from LOC scenarios in transport class aircraft. EA-FMS and iReCoVeR have been developed in parallel, with EA-FMS placing greater emphasis on control authority switching logic and iReCoVeR placing greater emphasis on adaptive control. The design of an aircraft guidance and control algorithm for actuator failure and structural damage was investigated by Chowdhary et al. [20].

\section{A. Envelope Estimation}

A flight envelope is a set of constraints. If these constraints are violated, LOC events become more likely [21]. Techniques to characterize aircraft states that can lead to future constraint adherence or violation are necessary to detect and resolve LOC events. This paper uses safe sets to plan constraint-admissible trajectories. A safe set $O_{\infty}$ is the set of aircraft initial states for which the nominal closedloop response does not violate the imposed constraints. The literature on computing safe sets and closely related forward and backward reachable sets for constrained aircraft dynamics is extensive; for example, see [22-29] and references therein. Many of the existing approaches exploit nonlinear models and require extensive computations to solve partial differential equations. Consequently, their onboard use for scenarios in which aircraft models must be identified online may be infeasible unless significant simplifications are made and short horizons are assumed [25]. 
Because the EA-FMS relies on identified linearized models, information about changes in parameters, such as $C_{D 0}$ and $C_{L 0}$, that impact the global flight envelope can be inferred from identified models [11]. These identified parameter changes can be used to update the flight envelope boundary constraints. Envelope constraint updates in turn change the state and control constraint representations used by a controller state and reference governor (CSRG) [9,30,31]. A CSRG resets internal controller states and the reference signal to improve performance and enforce the imposed state and control constraints based on the safe sets definition for this closed loop system: the set of all initial plant states, internal controller states, and references, such that the ensuing trajectories are constraint admissible. These sets are calculated for each equilibrium or trim condition based on linear models. They can be sequenced to ensure transitions between trim conditions are constraint admissible.

\section{B. Adaptive Flight Planner}

LOC avoidance and recovery require flight planning and guidance commands that keep the aircraft within a potentially reduced flight envelope space. Adaptive flight planner in EA-FMS may require functionality ranging from generating a single new reference state, as in climb reduction for stall avoidance, to a fully updated emergency landing flight plan. For the dangerous rudder-jam scenario, the EAFMS flight planner proposes a short-term emergency landing plan respecting degraded flight envelope constraints. The first task in emergency landing planning is to determine a suitable landing site(s). The flight planner then rapidly creates feasible paths to the selected site(s).

Airport runways are favored as landing sites. Airport database and weather information can inform a utility function to prioritize runways [14] with recent extension to consider off-runway landing sites if necessary [10]. Related work [32] combines en route and landing site risk in landing site prioritization at the cost of increased computational overhead.

Path or trajectory planning has been performed with fast geometric, as well as search and numerical optimization, methods. Geometric path planners for fixed-wing aircraft are typically three-dimensional (3-D) extensions of Dubins' minimum-length two-dimensional (2-D) path with a minimum turn radius constraint [33]. The third verticaldimension path can be specified independently of the planar motion with flight path angle limitations [34] or as a direct extension of the Dubins formulation, as in [35]. Minimum-length geometric solutions are typically linked with airplane kinodynamics constraints using fixed airspeed and bank angle values with small angles assumptions [36].

A segmented path can be constructed from trim state segments. Turning and straight segments can be connected using results derived from geometric planners such as Dubins [14]. Such path planners respect airplane dynamic constraints in each segment, and state transitions can also be taken into consideration in complete solutions; for example, see $[37,38]$ and references therein.

Generalized path planning with dynamic constraints can be performed using optimal control. Initial and final states define a twopoint boundary value problem (BVP). Several methods have been developed, yet high computational overhead and strong dependence of solution convergence on boundary points make such solvers impractical for real-time use [39]. Optimal control performance can be improved by computing reference trajectories offline and refining them online as in neighboring optimal control [40]. Optimal control can also be used to time-parameterize geometric paths [39], and receding horizon approaches [41] offer short-term solutions that improve as a function of available computational resources. Optimality criteria must always be clearly specified. For nominal flight, balanced minimization of fuel and time is appropriate. For emergency landing, fuel and time are not as critical as safety, both of the selected landing site and of the path to that site. Such criteria therefore require modification to traditional optimality criteria, as discussed in this work.

\section{Flight Safety Assessment and Management}

FSAM serves as the activation/deactivation switch for the envelopeaware FMS. FSAM monitors flight conditions for anomalies and assesses risks associated with the current flight conditions. If the flight crew does not respond with appropriate control actions in time to assure recovery, FSAM overrides with envelope-aware control to ensure LOC risk is mitigated. FSAM relies on aircraft state and control input information, quantitative and qualitative metrics obtained from the envelope estimation, system identification, and flight planning modules to make override decisions. Techniques that use linear temporal logic, finite state machines, flow charts, reachable sets, and Markov decision processes (MDP)/partially observable MDP have been previously used to construct decision-making logic similar to FSAM. Chongvisal et al. [18] developed an LOC prediction and prevention system that used manually defined thresholds to monitor flight state relative to quantitative LOC envelopes developed by Wilborn and Foster [21]. An envelope protection control law was activated if the aircraft violated the thresholds and would then return the aircraft back into the safe operating regime. However, this work did not consider the effect of changing flight envelopes. Control mode switching was proposed by Tomlin et al. [42] based on reachable sets for collision avoidance. Manually engineered flow charts were used by Gingras et al. [16] and Srivatsan et al. [43]. The decision variables in these flow charts were based on aerodynamic and flight performance look-up tables. These systems only focused on warning the flight crew about imminent LOC situations. A decision theoretic approach using MDPs was proposed by Kochenderfer and Chryssanthacopoulos [44] in an updated traffic collision avoidance system (TCAS). Both TCAS and Runway Overrun Protection System (ROPS) [45] are commercially available today. Most provide warnings to the crew but do not override crew authority to ensure timely recovery. The Automatic Ground Collision Avoidance System (Auto-GCAS) developed by the U.S. Air Force Research Laboratories [46] prevents controlled flight into terrain (CFIT) situations. Using prior maps, a trajectory-prediction routine and a collision-avoidance routine the Auto-GCAS system overrides the pilot with a recovery autopilot to prevent imminent CFIT. The AutoGCAS system is consistent with the ideals of FSAM.

Previous publications introduced and developed FSAM as a highlevel decision-making module that can address LOC issues related to aircraft flight dynamics and control, aircraft health, and pilot and environment characteristics. A general FSAM system formulated as an MDP was provided in [7,47]. Specialization of FSAM to prevent LOC events associated with takeoff was presented in $[\underline{5}-\underline{7}, \underline{48}]$.

\section{Envelope Estimation and the Controller State and Reference Governor}

This paper models the global flight envelope as a collection of trim conditions the aircraft can maintain in steady-state. A trim condition is an equilibrium of the nonlinear aircraft dynamics corresponding to constant actuator inputs. Flight envelope boundaries are defined by the minimum and maximum airspeed, angle of attack, sideslip, attitude, and angular rates (see Appendix A).

The availability of stabilizable trim conditions within the steadyflight envelope enables the development of control laws based on linearized aircraft dynamics at these trim conditions. The linear controllers are only valid in a neighborhood around the corresponding trim conditions. Safe sets are used to characterize constraintadmissible deviations from the trim condition and to determine if constraint-admissible transitions between the trim conditions can be performed.

\section{A. Safe Sets and Safe Trim Transition Sequence Estimation}

Aircraft controllers typically use integral action to guarantee offset-free constant reference command tracking and asymptotic constant input disturbance rejection. Suppose closed-loop system dynamics can be locally (near a selected trim condition) represented by the following linear model,

$$
\begin{gathered}
\bar{x}(k+1)=\bar{A} \bar{x}(k)+\bar{B} \bar{r}(k), \\
\bar{y}(k)=\bar{C} \bar{x}(k)+\bar{D} \bar{r}(k)
\end{gathered}
$$

in which $\bar{r}(k)$ is a reference. The state $\bar{x}(k)=\left[x^{T}, x_{I}^{T}\right]^{T}$ is composed of nonresettable components $x$, which correspond to the deviations of 
the aircraft states from the trim condition, and of resettable components $x_{I}$, which correspond to the integrator dynamics in the controller of the form

$$
x_{I}(k+1)=x_{I}(k)+C_{I} x(k)-\bar{r}(k)
$$

The matrix $\bar{A}$ is assumed to be Schur (i.e., all eigenvalues are within the unit circle), and we let $\bar{x}_{e}=H \bar{r}$ denote the equilibrium state of the linear model corresponding to a constant reference $\bar{r}(k) \equiv \bar{r}$. The nominal reference is denoted by $\bar{r}^{*}$. If $\bar{r}^{*}=0, \bar{x}$ converges to the origin. Control, model validity, and safety constraints lead to local constraints on the output of Eq. (1) of the form

$$
\bar{y}(k) \in \bar{Y}=\{\bar{y}: G \bar{y} \leq g\}
$$

Constraints from the presented case study are discussed in Appendix A. To characterize safe states and references, we introduce the following safe set,

$$
\begin{aligned}
& O_{\infty}^{r}=\{(\bar{x}(0), \bar{r}):(\bar{C} H+\bar{D}) \bar{r} \in(1-\epsilon) \bar{Y} \\
& \quad \text { and } \quad r(k)=\bar{r} \quad \forall k \geq 0 \Rightarrow \bar{y}(k) \in \bar{Y} \quad \forall k \geq 0\},
\end{aligned}
$$

in which $\bar{y}(k)=\left(\bar{C}\left(I-\bar{A}^{k}\right)(I-\bar{A})^{-1} \bar{B}+\bar{D}\right) \bar{r}+\bar{C} \bar{A}^{k} \bar{x}(0)$ and in which $\epsilon>0$ is sufficiently small. $O_{\infty}^{r}$ is the set of all initial states $\bar{x}(0)$ and constant reference signals $\bar{r}$ that lead to responses satisfying the constraints. If $\bar{A}$ is Schur $(\bar{C}, \bar{A})$ is observable, $0 \in \operatorname{int} \bar{Y}$, and $\bar{Y}$ is compact, $O_{\infty}^{r}$ in Eq. (4) is positively invariant, finitely determined (i. e., represented by a finite number of affine inequalities), convex, and is a polytope [49]. To guarantee $O_{\infty}^{r}$ is finitely determined, the set of admissible constant reference signals is tightened slightly from $(\bar{C} H+\bar{D}) \bar{r} \in \bar{Y}$ to $(\bar{C} H+\bar{D}) \bar{r} \in(1-\epsilon) \bar{Y}$.

The safe sets can be used in aircraft flight planner to determine a sequence of trim conditions that can be safely followed by the aircraft. Let $X$ denote the vector of (nonresettable) aircraft states, and let $X_{\mathrm{eq}}^{n}$ denote the vector of states at the $n$th trim condition so that $x=X-X_{\mathrm{eq}}^{n}$. The condition for connectivity and feasibility of a safe (constraint-admissible) transition from trim condition $X_{\mathrm{eq}}^{n}$ to trim condition $X_{\mathrm{eq}}^{n+1}$ can be expressed as

$$
X_{\mathrm{eq}}^{n}-X_{\mathrm{eq}}^{n+1} \in \operatorname{int} R_{\infty}^{n+1}
$$

in which $R_{\infty}^{n+1}$ denotes the projection of the safe set $O_{\infty}^{r}=O_{\infty}^{r, n+1}$, corresponding to the linearized closed-loop model at the $(n+1)$ st trim condition $X_{\text {eq }}^{n+1}$, onto the aircraft states $x$.

The actual reset of the controller states and adjustment of the reference is performed by the CSRG described in the next subsection. With $\bar{r}^{*}=0$, the CSRG convergence results guarantee $\bar{r}(k) \rightarrow 0$ and aircraft states converging asymptotically to the currently commanded trim condition, $X_{\mathrm{eq}}^{n}$ in the sequence. With the connectivity between different trim conditions established based on Eq. (5), a graph search can be performed to determine a path between the origin and destination trim conditions. Our approach in this paper is distinguished (e.g., versus [50] and references therein) by exploiting sets $R_{\infty}$ (which are projections of safe sets) and by the reset of controller states and references as necessary to effect constraint-admissible transitions.

Feasibility of transitions between certain trim conditions is a function of rudder-jam angle in this paper's case study. Equation (5) is used to compute connectivity graphs that identify feasible transitions between trim conditions. Although other implementations are possible, in this paper, these connectivity graphs are computed offline for different jam angles and stored in a database that can be accessed in real time to plan a constraint-admissible flight plan to a safe landing site. It is straightforward to make the switching sequence robust against the deviations of the jammed rudder away from discrete values used in the mesh by modifying the definition of $O_{\infty}^{r}$ to account for constant or bounded time-varying disturbances due to the deviations.

\section{B. Controller State and Reference Governor}

The CSRG is used to execute constraint-admissible transitions between the trim conditions. The CSRG resets $x_{I}(k)$ and determines $\bar{r}(k)$ based on constrained minimization of the cost function,

$$
J(\bar{x}(k), \bar{r}(k))=\left\|\bar{x}(k)-H \bar{r}^{*}\right\|_{P}^{2}+\left\|\bar{r}(k)-\bar{r}^{*}\right\|_{\Gamma}^{2}
$$

in which, for a matrix $M,\|z\|_{M}^{2}=z^{T} M z, \Gamma=\Gamma^{T}>0$, and $P=$ $P^{T}>0$ satisfies the Lyapunov equation $P=\bar{A}^{T} P \bar{A}+Q_{L}$ with $Q_{L}=Q_{L}^{T}>0$. To determine $x_{I}(k)$ and $\bar{r}(k)$, quadratic programming problem $J(\bar{x}(k), \bar{r}(k)) \rightarrow \min _{x_{I}(k), \bar{r}(k)}$ is solved subject to $(\bar{x}(k), \bar{r}(k))$ $\in O_{\infty}^{r}, \bar{x}(k)=\left[x(k)^{T}, x_{I}(k)^{T}\right]^{T}$. Note that $x(k)=X(k)-X_{\mathrm{eq}}^{n}$ is the current deviation of the aircraft state from the trim condition $X_{\mathrm{eq}}^{n(k)}$, which is currently being commanded. CSRG convergence results, including $\bar{r}(k) \rightarrow \bar{r}^{*}$ and $\bar{x}(k) \rightarrow H \bar{r}^{*}$, are derived in [30,31]. Feasibility of this optimization problem configured around a given trim condition implies that a constraint-admissible transition to that trim condition is feasible by appropriate reset of the controller state and reference.

\section{Adaptive Flight Planner}

Adaptive flight planner (AFP) uses the estimated envelope to choose a goal waypoint and associated flight plan. For safety-critical failure cases such as rudder jam, the AFP builds an emergency landing plan by first identifying a suitable landing site and then constructing a path to it. The AFP follows the two-step process proposed in [14]. A landing-site search (LSS) function first identifies and prioritizes suitable runways, and an extended final Dubins path planner (EF-DPP) then builds a trim state path to the highest-ranked suitable runway. Integration with envelope estimation requires trim condition preprocessing (TCP) offline to translate the envelope estimate to a tractable trim state set to be used by the trajectory planner. TCP, LSS, and EF-DPP are described below.

\section{A. Trim Condition Preprocessing (TCP)}

For any anomaly or failure that can be characterized a priori, envelope estimation can be performed offline. For a rudder jam, analysis can occur over a family of jam angles to ensure results are applicable to any specific case online. The envelope can be converted to a graph in which nodes are trim conditions and edges connect neighboring trim conditions that can be accessed without envelope violations. Because not all trim conditions are mutually reachable, the graph is not fully-connected. Graph nodes and edges may also vary as a function of altitude. Recall that optimality for emergency landing is driven by flight safety rather than metrics such as fuel.

Different schemes can be employed to construct the envelope. This work defines valid trim conditions for flight planner as those that can be reached from a wings-level constant altitude or cruise trim state. This is a compromise between checking all possible transitions between trim states in the database and only checking the immediate neighbor trim states. Such sets of valid trim conditions are constructed for each pair of altitude and airspeed. In this work, airspeed is held constant, and the intersection of sets of feasible trim conditions for each altitude is used as an overall feasible set of trim conditions.

Once safe trim conditions and transitions are identified, groups of four are created and prioritized for the Dubins-based path planner described below. To downselect from the $O\left(n^{4}\right)$ possible sequences of the $n$ feasible trim states, airspeed is held constant at its initial trim value. In future work, sequencing states with progressively lower airspeeds would assist in minimizing the energy to dissipate after runway touchdown. Trim states at the borders of the trim state database and connected trim state space are discarded to provide a margin for guidance. Trim conditions are ordered as follows for the Dubins solution described below: The first and third trim condition must be turning flight with preference for tighter turns and steeper descent rates. The second trim condition is straight flight, with preference given to steeper descent rates, whereas the fourth final approach trim condition must be straight, with a preferred flight path angle of $-3 \mathrm{deg}$ or as close to that value as possible. 
For the rudder-jam scenario TSP can be performed offline, allowing a human operator to verify the reduced trim condition set(s) are appropriate choices. Note that the selected steeper descent rates correspond to approximately a 4-deg descent flight path angle that is well within the feasible envelope; a steep descent shortens the path extensions required to manage altitude.

\section{B. Landing-Site Search (LSS)}

LSS is the process of finding and ranking one or more feasible landing sites, ideally as a safe runway. The rudder-jam case does not impose hard constraints on flight time or range. Therefore, instead of calculating a footprint as in [14], this work simply assumes a fixed footprint radius in which candidate landing runways are identified. Runway information is acquired from a Federal Aviation Administration database of U.S. airports [51]. The full database was preprocessed to filter for data relevant to LSS, resulting in a small $(\sim 600 \mathrm{kB})$ data file that would be loaded for real-time use on the airplane. Reachable runways are ordered according to the following utility-based function, as an extension to [14]:

$$
\begin{aligned}
& U=\sum_{i} C_{i} w_{i}=C_{1} \frac{r_{l}}{r_{l, \max }}+C_{2} \frac{r_{w}}{r_{w, \max }}+C_{3} q_{i} \\
& +C_{4} \frac{\mathrm{d}}{\mathrm{d}_{\max }}+C_{5} \frac{\left(w_{h}-w_{h, \min }\right)}{\left(w_{h, \max }-w_{h, \min }\right)}+ \\
& +C_{6} \frac{\left(w_{c, \max }-w_{c}\right)}{\left(w_{c, \text { max }}-w_{c, \text { min }}\right)}+C_{7} q_{S}+C_{8} q_{\mathrm{nf}}+C_{9} c_{\mathrm{ATC}}+C_{10} c_{\mathrm{ff}}
\end{aligned}
$$

Ten parameters are included in this function: runway length $r_{l}$ relative to maximum available runway length $r_{l \text {,max }}$, runway width $r_{w}$ relative to maximum available runway width $r_{w, \max }$, instrument approach quality $q_{i}$, distance $d$ relative to maximum landing site search radius $d_{\max }$, headwind velocity $w_{h}$, crosswind velocity $w_{c}$, surface quality $q_{s}$, resources for the next flight availability measure $q_{\mathrm{nf}}$, staffed air traffic control tower availability $q_{\text {atc }}$, and firefighter protection availability $q_{\mathrm{ff}}$. The last two terms are new to this work. As originally proposed [14], parameters are normalized so that each factor of the utility function changes from 0 to 1 . Note that, for wind terms, a special normalization is needed. For this utility term, crosswind component $w_{c}$ is always considered positive, which requires use of the crosswind magnitude without consideration of left/right direction. Additionally, tailwinds must also be included in the $w_{h}$ term. The proposed formula subtracts the maximum tailwind (or minimum headwind $w_{h \text {, min }}$ ) over all reachable runways to ensure this utility term is nonnegative. Details of $q_{i}, q_{s}, q_{\mathrm{nf}}, q_{\mathrm{atc}}$ and $q_{\mathrm{ff}}$ are presented in Appendix B. Note that the weights $C_{i}$ can be chosen by pilots or airliners for different failure conditions according to their preference.

\section{Extended Final Dubins Path Planner (EF-DPP)}

The baseline AFP formulation [14] generates segmented paths for selected runways. Those trajectories are Dubins paths, an extension of Dubins seminal work [33] defining turning-constrained minimumlength 2-D paths. In previous work [14], a range of feasible flight path angles was defined, with the solution adopting the midpoint to maximize margins. This assumption allows the 2-D Dubins curve defining the lateral path to be combined with an altitude profile that can be adjusted in the plan and during trajectory tracking. When a finite set of trim conditions is available, altitude adjustment requires an additional control parameter, such as landing path length.

Given the current airplane position and the location of the goal state at the location and heading of the chosen runway threshold, the first step is to create two waypoints. Waypoint 0 is a certain distance ahead [1.0 nautical miles (NM) for this work] of the current position at the current heading with altitude hold; waypoint 0 serves as the initial planner state, providing time between plan generation and activation. Moreover, flight time up to waypoint 0 can be used by the flight crew to check the proposed flight plan and goal airport and make changes if desired. Waypoint 4 is created at a fixed final approach leg distance from the runway threshold (1.5 NM in this work) at an altitude corresponding to a 3-deg final approach flight path

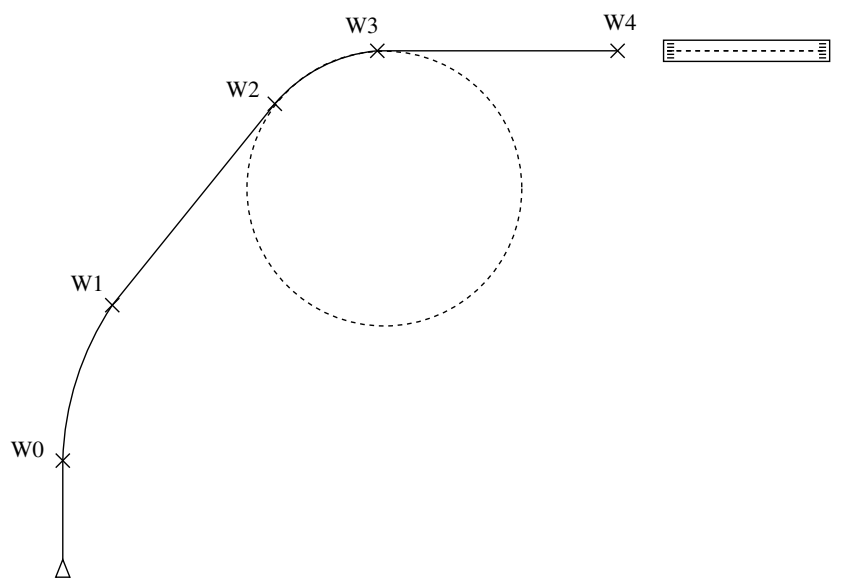

Fig. 2 Extended final Dubins path planner.

angle at runway heading. The path planner must then connect waypoints 0 and 4 . This work uses segmented trajectories composed of four segments, two straight segments and two turns, generating an "extended final trajectory" as described in [14]. Segment length is obtained using a tractable hill-climbing algorithm. If the required altitude loss is sufficiently large, complete 360-deg turns are included in the second turn segment as necessary. Figure 2 illustrates the waypoint numbering, and Algorithm 1 presents the algorithm to calculate waypoints $1-3$, given a set of trim conditions.

In Algorithm $1,\left(x_{i}, y_{i}, z_{i}, \sigma_{i}\right)$ are the position and heading associated with waypoint $\bar{W}_{i}$, and $S=\left\{\left(R_{i}, \gamma_{i}\right) \mid 0 \leq i \leq 3\right\}$ is the set of trim conditions defined by TSP. Note that each trim state $i$ is translated to a turn radius $R_{i}$ and a flight path angle $\gamma_{i} . \eta$ is the distance between waypoints 3 and 4 , and $n_{\text {turns }}$ is the number of complete 360 -deg turns in the trajectory. PositionFromADistanceGivenPathAngle calculates a position relative to an initial position given a distance, heading, and flight path angle, whereas Dubins is a 2-D Dubins solver which uses two different turn radii. This paper only considers turn-straight-turn solutions that are possible in most cases, including the presented case study. Also note that the precomputed trim states determine the direction of each turn. CalculateAltitudes determines the altitudes for each waypoint given the path distance between them and the flight path angles associated with the specified trim states. Although this algorithm restricts the possible trajectories to those composed of two straight and two turning segments, the solver can run in real time over multiple sets of trim conditions and runways. This capability also mitigates eventual problems in case the solver cannot find a solution for a certain runway with a certain set of trim states. The solver cannot find a solution, for example, if there is not enough altitude ( $\eta$ becomes negative), Dubins solution does not exist or iterations exceed a predetermined number.

\section{Flight Safety Assessment and Management}

The FSAM module of EA-FMS constantly monitors flight conditions to assess LOC risk. Under high-risk situations, FSAM can override the nominal automation to mitigate the LOC risk. FSAM

\section{Algorithm 1 Extended Final Dubins Path Planner}

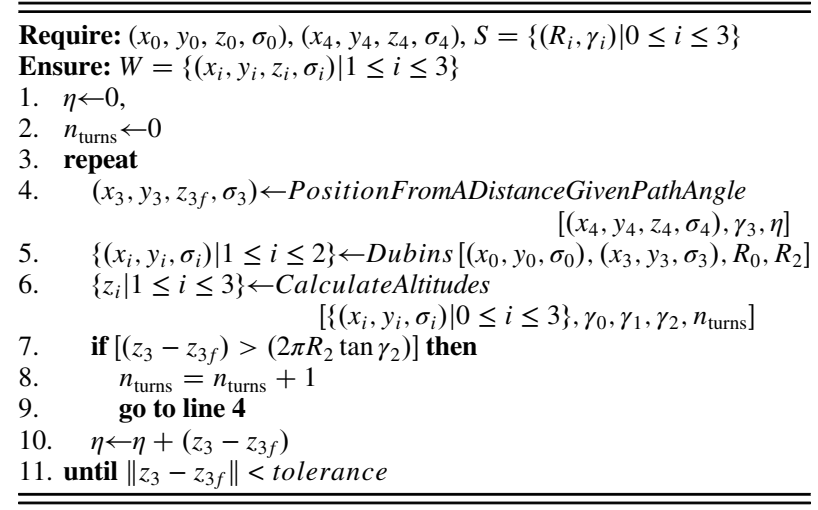


effectively serves as an "LOC watchdog" that generalizes specific override modules such as flight envelope protection. Formulating FSAM over all possible LOC scenarios is challenging. As an initial simplification step, each flight is first decomposed by phase, including takeoff, climb, cruise, descent, approach, and landing. Within each phase, FSAM can be further decomposed to address LOC scenarios associated with off-nominal conditions such as actuator malfunction, sensor and instrument failures, and their combinations. The suite of FSAM submodules for each flight phase simultaneously monitor for the LOC risk(s) they address. FSAM can be implemented as a deterministic state machine (e.g., Moore machine) with output at each state prescribing any control authority switching based on understanding of LOC events and mitigation strategies [6]. A state machine formulation facilitates verification, validation, and certification of the underlying logic. However, manually designing these finite state machines to address all possible LOC scenarios has substantial workload and requires a priori specification of all parameters governing risk assessment and FSAM authority switching.

In this work FSAM is formulated as an $\operatorname{MDP}(\mathcal{S}, \mathcal{A}, \mathcal{T}, \mathcal{R})$, in which $\mathcal{S}$ represents a finite state set, $\mathcal{A}$ represents a finite action set, $\mathcal{T}: \mathcal{S} \times \mathcal{A} \times \mathcal{S} \rightarrow[0,1]$ represents state transition probabilities, and $\mathcal{R}: \mathcal{S} \times \mathcal{A} \rightarrow \mathbb{R}$ represents a reward function that assigns a finite real value to each state-action pair. Actions $a_{n} \in \mathcal{A}$ for each state $s_{n} \in \mathcal{S}$ at each decision epoch are chosen such that they maximize the expected cumulative discounted reward. A general MDP formulation for FSAM was provided in our complementary work [ 7,47$]$. The optimal policy $\pi: \mathcal{S} \rightarrow \mathcal{A}$ prescribes the optimal action to execute at each state. To select the appropriate control authority for LOC recovery, the FSAM MDP [7,47] state features describe aircraft dynamics and control, aircraft health, pilot state, and environment characteristics. The MDP actions enable FSAM to remain a passive monitor under low-to-moderate-risk conditions while overriding the nominal pilot/autopilot system with the envelope-aware controller under high-risk conditions. The reward function penalizes unsafe states and unnecessary changes in control authority. For simplicity, this work assumes that the features related to the pilot and environment remain constant at nominal low-risk values. In this work, the MDP state space is compactly described as

$$
s=[\bar{V}, \bar{A}, \bar{\Theta}, \bar{\Phi}, \bar{D}, \bar{M}, \bar{S}]
$$

Here, $\bar{V} \in\left\{\bar{v}_{1}, \bar{v}_{2}, \bar{v}_{3}, \bar{v}_{4}, \bar{v}_{5}, \bar{v}_{6}\right\}$ represents proximity to the airspeed envelope boundaries with $\bar{v}_{1}=\left\{V \mid V \leq V_{\min }\right\}, \bar{v}_{2}=\{V \mid 0<$ $\left.\left(V-V_{\min }\right) \leq 0.1 \Delta V\right\}, \quad \bar{v}_{3}=\left\{V \mid 0.1 \Delta V<\left(V-V_{\min }\right) \leq 0.4 \Delta V\right\}$,
$\bar{v}_{4}=\left\{V \mid 0.4 \Delta V<\left(V-V_{\min }\right) \leq 0.9 \Delta V\right\}, \bar{v}_{5}=\left\{V \mid 0.9 \Delta V<\left(V-V_{\min }\right) \leq\right.$ $\Delta V\}$, and $\bar{v}_{6}=\left\{V \mid V \geq V_{\max }\right\}$. Note that $V=\sqrt{u^{2}+v^{2}+w^{2}}$ represents the airspeed. $V_{\min }$ and $V_{\max }$ represent minimum and maximum airspeeds, respectively. $\Delta V=V_{\max }-V_{\min }$. Note that aircraft stall occurs in $\bar{v}_{1}$ and is an unsafe airspeed state. $\bar{v}_{6}$ poses high structural-damage risk due to aerostructural forces and moments.

$\bar{A} \in\left\{\bar{\alpha}_{1}, \bar{\alpha}_{2}, \bar{\alpha}_{3}\right\}, \bar{\alpha}_{i}=\left\{X \mid A_{i} X \leq B_{i}\right\}$ represents proximity to the adverse aerodynamic envelope boundaries introduced by Wilborn and Foster [21]. $\bar{\alpha}_{1}, \bar{\alpha}_{2}$ and $\bar{\alpha}_{3}$ represent partitions of the adverse aerodynamic envelope. $X=\left[\alpha_{m}, \beta_{m}\right]$, in which $\alpha_{m}$ and $\beta_{m}$ represent the normalized angle of attack and sideslip angles respectively. $A_{i}$ and $B_{i}$ are constraint matrices chosen appropriately to represent the envelope boundaries as shown in Fig. 3 .

$\bar{\Theta} \in\left\{\bar{\theta}_{1}, \bar{\theta}_{2}, \bar{\theta}_{3}, \bar{\theta}_{4}, \bar{\theta}_{5}, \bar{\theta}_{6}\right\}, \quad \bar{\theta}_{i}=\left\{\bar{X} \mid A_{i} X \leq B_{i}\right\}$ is a compact representation of aircraft pitch $\theta$, pitch rate $q$, and elevator control $\delta_{e}$. Specifically, $\bar{\Theta}$ is a discretization of the dynamic pitch control envelope introduced by Wilborn and Foster [21]. The dynamic pitch $\theta^{\prime}$ is defined as $\theta+q . \bar{\Theta}$ is defined below and also illustrated in Fig. 3. Here, $X=\left[\theta^{\prime}, \delta_{e}\right]$. $\bar{\theta}_{1}, \ldots, \bar{\theta}_{6}$ represent partitions in the dynamic pitch control envelope (see Fig. $\underline{3}$ ) and can be expressed as linear constraints as shown above. As illustrated in [21], $\bar{\theta}_{1}$ and $\bar{\theta}_{2}$ represent safe operating envelope regions. $\bar{\Phi} \in\left\{\bar{\phi}_{1}, \ldots, \bar{\phi}_{6}\right\}$ is a compact representation of aircraft roll $\phi$, pitch rate $p$, and aileron control $\delta_{a}$ and can be defined similar to $\bar{\Theta}$. Dynamic roll $\phi^{\prime}$ is defined as $\phi+p . \bar{\Phi}$ is a discretization of the dynamic roll control envelope in [21]

$\bar{D} \in\left\{\bar{d}_{1}, \bar{d}_{2}, \bar{d}_{3}\right\}$ characterizes deviation from the current flight plan and is defined based on cross track deviation from the current flight plan and the current aircraft heading, as illustrated in Fig. $\underline{3}$. Similar to $\bar{\Theta}$ and $\bar{\Phi}, \bar{D}$ can also be defined by linear inequalities. This work defines two control modes $\bar{M} \in\{P, \mathrm{EA}\}$, in which $P$ represents the pilot in control mode and EA represents the envelope-aware control mode. $\bar{S} \in\{P, \mathrm{EA}\}$ denotes the mode select switch that the pilot could use to manually activate the EA controller or to request control back from the EA controller.

The compact state representation does not include rudder actuator health status. Because a family of MDP policies is constructed and executed in accordance with specific failure condition, the MDP constructed for this paper's rudder-jam case study presumes the rudder has failed and will remain failed throughout policy execution.

The state features discussed above are functions of envelope parameters. These parameters can be stored for different anomaly cases a priori or updated online via envelope estimation. The proposed state-

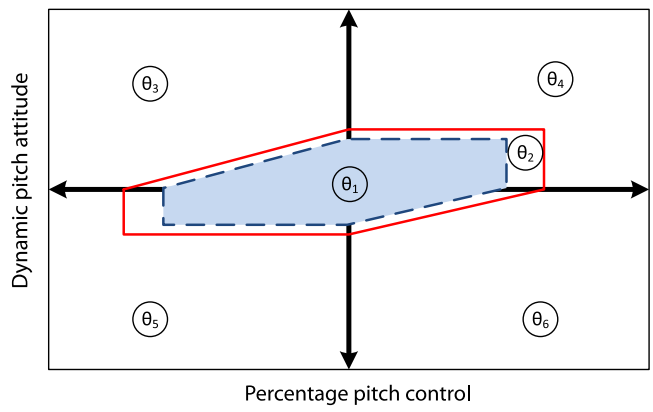

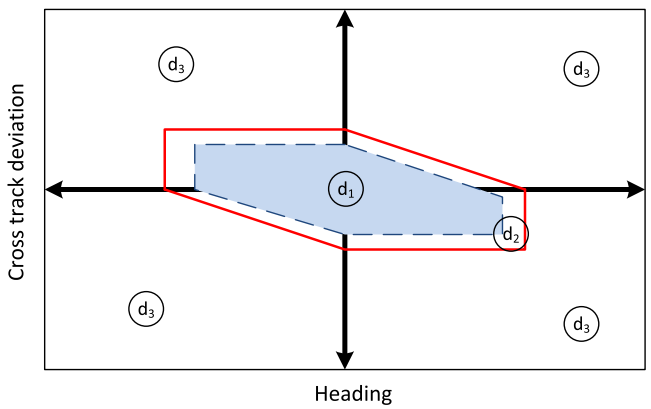

Percentage lateral control

(4)

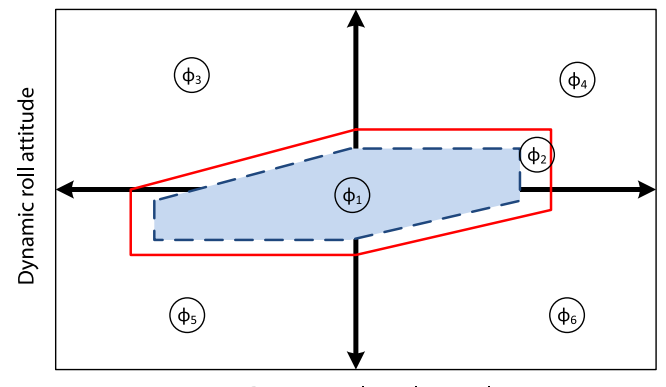

Fig. 3 State space partitions: adverse aerodynamic envelope (top left); dynamic pitch control (top right), dynamic roll control (bottom left), and flight plan deviation (bottom right). 
space abstraction is sufficient for the rudder-jam case study considered in this work, as well as for other risk scenarios that can be modeled with the dynamic envelope structure proposed by Wilborn and Foster [21]. For FSAM, the MDP actions are $a \in\{$ NOOP, TOGL $\}$ as in [7]. NOOP (no operation) is chosen under nominal conditions in which FSAM monitors for risk and under conditions in which envelopeaware authority must persist. TOGL (toggle) overrides the current control authority to either activate envelope-aware control or restore control to the crew. State transition probabilities can be obtained via Monte Carlo simulations. An additive reward $\mathcal{R}(s, a)=\sum_{i} w_{i} \mathcal{R}_{i}$ is defined in which the $\mathcal{R}_{i}$ penalize unsafe states, as shown below and the $w_{i}$ are weighting parameters.

$$
\begin{aligned}
& \mathcal{R}_{1}=\left\{\begin{array}{cc}
-1 & \text { if } \bar{V} \in\left\{\bar{v}_{1}, \bar{v}_{6}\right\} \\
0 & \text { otherwise }
\end{array} \quad \mathcal{R}_{2}=\left\{\begin{array}{cc}
-1 & \text { if } \bar{A} \in\left\{\bar{\alpha}_{3}\right\} \\
0 & \text { otherwise }
\end{array}\right.\right. \\
& \mathcal{R}_{3}=\left\{\begin{array}{cc}
-1 & \text { if } \Theta \in\left\{\bar{\theta}_{3}, \bar{\theta}_{4}, \bar{\theta}_{5}, \bar{\theta}_{6}\right\} \\
0 & \text { otherwise }
\end{array} \quad \mathcal{R}_{4}=\left\{\begin{array}{c}
-1 \\
0 \text { if } \Phi \in\left\{\bar{\phi}_{3}, \bar{\phi}_{4}, \bar{\phi}_{5}, \bar{\phi}_{6}\right\} \\
\text { otherwise }
\end{array}\right.\right. \\
& \mathcal{R}_{5}=\left\{\begin{array}{cc}
-1 & \text { if } \bar{D} \in\left\{\bar{d}_{3}\right\} \\
0 & \text { otherwise }
\end{array}\right. \\
& \mathcal{R}_{6}=\left\{\begin{array}{cc}
-1 & \text { if } \bar{M}=P \wedge \bar{S}=P \wedge a=\mathrm{TOGL} \\
-o_{1} & \text { if } \bar{M}=\mathrm{EA} \wedge \bar{S}=P \wedge a=\mathrm{NOOP} \\
-o_{2} & \text { if } \bar{M}=\mathrm{EA} \wedge \bar{S}=\mathrm{EA} \wedge a=\mathrm{TOGL} \\
0 & \text { otherwise }
\end{array}\right.
\end{aligned}
$$

Here, $0 \leq o_{1} \leq 1$ and $0 \leq o_{2} \leq 1$. Note that $\mathcal{R}_{6}$ discourages frequent TOGL actions. Persistence in envelope-aware control mode is also penalized to encourage transfer of authority to the crew once the high-risk condition is mitigated. Weighting factors in these reward formulations are set to $w_{1}=100, w_{2}=100, w_{3}=50, w_{4}=50$, $w_{5}=50$, and $w_{6}=10$ and to $o_{1}=0.5$ and $o_{2}=1$ for the policy example in Table 1 also used for the rudder-jam study. The compact state features discussed above lead to a smaller state space. For cases such as rudder jam that can be analyzed a priori, optimal policies can be obtained offline using value iteration [52] and stored as look-up tables. The total number of states in the look-up table is given by the product of the size of the individual state features, which yields 7776 states. Selective content for a sample policy is shown in Table 1 . For this example, when the pilot is in control $(\bar{M}=P)$ and airspeed is in the safe operating region $\bar{V} \in\left\{\bar{v}_{3}, \bar{v}_{4}\right\}$, FSAM chooses NOOP (lines 3 and 4 in Table 1$)$. When the airspeed decreases $\left(\bar{V}=\bar{v}_{2}\right)$ to high-risk levels, TOGL activates envelope-aware control (line 2). If envelopeaware control is active $(\bar{M}=\mathrm{EA})$ and the airspeed is safe, the example policy executes TOGL to transfer control back to the pilot (line 15). This is due to $R_{6}$ in Eq. (12). Similar behavior is illustrated when approaching unsafe pitch and roll states.

Table 1 shows a policy excerpt when $\bar{S}=P$. Presuming the system is initialized with $M=P$, it will remain in mode $P$ until either FSAM executes a TOGL action or the crew manually requests activation of the envelope-aware controller with the mode select switch (i.e. setting $\bar{S}=$ EA). In this work, whenever $\bar{S}=\mathrm{EA}$, it is assumed that the crew desires EA control so that FSAM does not override this decision. For a rudder-jam scenario, excursions from safe partitions of the adverse aerodynamic, dynamic pitch control, or dynamic roll control envelopes can trigger TOGL to envelope-aware control as shown in Table 1 (lines 5-11). The probability of transitioning back into a lower risk state in the FSAM MDP is higher with envelope-aware control, and so this mode persists, although in future work the logic should be extended to TOGL back to $\bar{M}=P$ under conditions when $\bar{S}=P, \bar{M}=\mathrm{EA}$, and EA-FMS fails to recover flight within the stable envelope after sufficient recovery time elapses. Automatic computation of sufficient recovery time for EA control is beyond the scope of this paper.
Table 1 Parts of MDP policy for $\bar{S}=P$

\begin{tabular}{llllllll}
\hline \hline Lines & $\bar{M}$ & $\bar{D}$ & $\bar{\Phi}$ & $\bar{\Theta}$ & $\bar{A}$ & $\bar{V}$ & $a$ \\
\hline 1 & $\mathrm{P}$ & $\bar{d}_{1}$ & $\bar{\phi}_{1}$ & $\bar{\theta}_{1}$ & $\bar{\alpha}_{1}$ & $\bar{v}_{1}$ & TOGL \\
2 & $\mathrm{P}$ & $\bar{d}_{1}$ & $\phi_{1}$ & $\bar{\theta}_{1}$ & $\bar{\alpha}_{1}$ & $\bar{v}_{2}$ & TOGL \\
3 & $\mathrm{P}$ & $\bar{d}_{1}$ & $\phi_{1}$ & $\bar{\theta}_{1}$ & $\bar{\alpha}_{1}$ & $\bar{v}_{3}$ & NOOP \\
4 & $\mathrm{P}$ & $\bar{d}_{1}$ & $\phi_{1}$ & $\bar{\theta}_{1}$ & $\bar{\alpha}_{1}$ & $\bar{v}_{4}$ & NOOP \\
5 & $\mathrm{P}$ & $\bar{d}_{1}$ & $\phi_{1}$ & $\bar{\theta}_{1}$ & $\bar{\alpha}_{2}$ & $\bar{v}_{3}$ & TOGL \\
6 & $\mathrm{P}$ & $\bar{d}_{1}$ & $\phi_{1}$ & $\bar{\theta}_{1}$ & $\bar{\alpha}_{2}$ & $\bar{v}_{4}$ & TOGL \\
7 & $\mathrm{P}$ & $\bar{d}_{1}$ & $\phi_{1}$ & $\bar{\theta}_{2}$ & $\bar{\alpha}_{1}$ & $\bar{v}_{3}$ & TOGL \\
8 & $\mathrm{P}$ & $\bar{d}_{1}$ & $\phi_{2}$ & $\bar{\theta}_{1}$ & $\bar{\alpha}_{1}$ & $\bar{v}_{3}$ & TOGL \\
9 & $\mathrm{P}$ & $\bar{d}_{1}$ & $\bar{\phi}_{1}$ & $\bar{\theta}_{2}$ & $\bar{\alpha}_{2}$ & $\bar{v}_{3}$ & TOGL \\
10 & $\mathrm{P}$ & $\bar{d}_{1}$ & $\phi_{2}$ & $\bar{\theta}_{1}$ & $\bar{\alpha}_{2}$ & $\bar{v}_{3}$ & TOGL \\
11 & $\mathrm{P}$ & $\bar{d}_{1}$ & $\phi_{2}$ & $\bar{\theta}_{2}$ & $\bar{\alpha}_{1}$ & $\bar{v}_{3}$ & TOGL \\
12 & $\mathrm{P}$ & $\bar{d}_{2}$ & $\phi_{1}$ & $\bar{\theta}_{1}$ & $\bar{\alpha}_{1}$ & $\bar{v}_{3}$ & TOGL \\
13 & EA & $\bar{d}_{1}$ & $\phi_{1}$ & $\bar{\theta}_{1}$ & $\bar{\alpha}_{1}$ & $\bar{v}_{1}$ & NOOP \\
14 & EA & $\bar{d}_{1}$ & $\phi_{1}$ & $\bar{\theta}_{1}$ & $\bar{\alpha}_{1}$ & $\bar{v}_{2}$ & NOOP \\
15 & EA & $\bar{d}_{1}$ & $\phi_{1}$ & $\bar{\theta}_{1}$ & $\bar{\alpha}_{1}$ & $\bar{v}_{3}$ & TOGL \\
16 & EA & $\bar{d}_{1}$ & $\bar{\phi}_{1}$ & $\bar{\theta}_{1}$ & $\bar{\alpha}_{1}$ & $\bar{v}_{4}$ & NOOP \\
17 & EA & $\bar{d}_{1}$ & $\bar{\phi}_{1}$ & $\bar{\theta}_{1}$ & $\bar{\alpha}_{1}$ & $\bar{v}_{5}$ & NOOP \\
18 & EA & $\bar{d}_{1}$ & $\phi_{1}$ & $\bar{\theta}_{1}$ & $\bar{\alpha}_{2}$ & $\bar{v}_{6}$ & NOOP \\
19 & EA & $\bar{d}_{1}$ & $\phi_{1}$ & $\bar{\theta}_{2}$ & $\bar{\alpha}_{1}$ & $\bar{v}_{3}$ & NOOP \\
20 & EA & $\bar{d}_{1}$ & $\phi_{2}$ & $\bar{\theta}_{1}$ & $\bar{\alpha}_{1}$ & $\bar{v}_{3}$ & NOOP \\
\hline \hline
\end{tabular}

\section{Data Management and Processing}

This section describes the offline and online steps required to prepare and execute EA-FMS for LOC scenarios that can be anticipated and modeled before in-flight occurrence. Examples of such failure cases are actuator jams and loss of engine thrust. LOC risk events that require online identification of the current dynamic model, such as airframe icing, must be modeled in flight and are considered in complementary work [53]. Analysis of LOC scenarios in advance is advantageous when possible, because offline modeling facilitates the validation and verification required for flight certification using current procedures.

\section{A. Offline Tasks}

Figure 4 shows the offline preprocessing required to prepare EAFMS for failure and other exception cases that can be accurately modeled in advance. First, the engineering team must specify an aircraft model that captures the failure/exception case under consideration. This model is used to create a database of possible trim (equilibrium) conditions that can be achieved for this case, for example, a rudder jammed at a particular angle.** The trim database is parametrized by airspeed, climb rate, turn rate, and altitude. Note that this trim database is one layer down from the top-level EA-FMS case database; that is, there will be one trim database per EA-FMS case. For each trim combination, the database entry contains the corresponding aircraft state, control surface deflections, and also corresponding linearized model parameters.

The safe sets at each trim condition are computed as described in Sec. III.A. With knowledge of the safe sets at each trim condition, a trim transition or connectivity graph is constructed. Trim conditions are represented as nodes while directed edges represent feasible transitions according to Eq. (5). The trim transition graph is then processed as described in Sec. IV.A to build sets of trim sequences to be used for realtime path planning. A suite of MDP policies for nominal and exception cases are computed offline per Sec. V. Figure 4 shows the process by which each MDP policy is created and stored. $\bar{B}$ y building the policies offline, each can be verified manually to ensure that control authority switching occurs when unsafe states are encountered. If the policy does not have the desired characteristics, determined manually and/or through formal verification, the weighting parameters in the MDP reward formulation [Eqs. (9-12)] are tuned to obtain the desired policy. This process of verifying the $\bar{M} D P$ policy can be intractable if the state space is too large. To facilitate policy verification in large MDPs, the probability of entering unsafe states can be constrained via the constrained MDP framework described in []].

**For control surface jams, each database case might handle an interval of jam angles so long as a conservative trim database applicable across the interval is identified. 


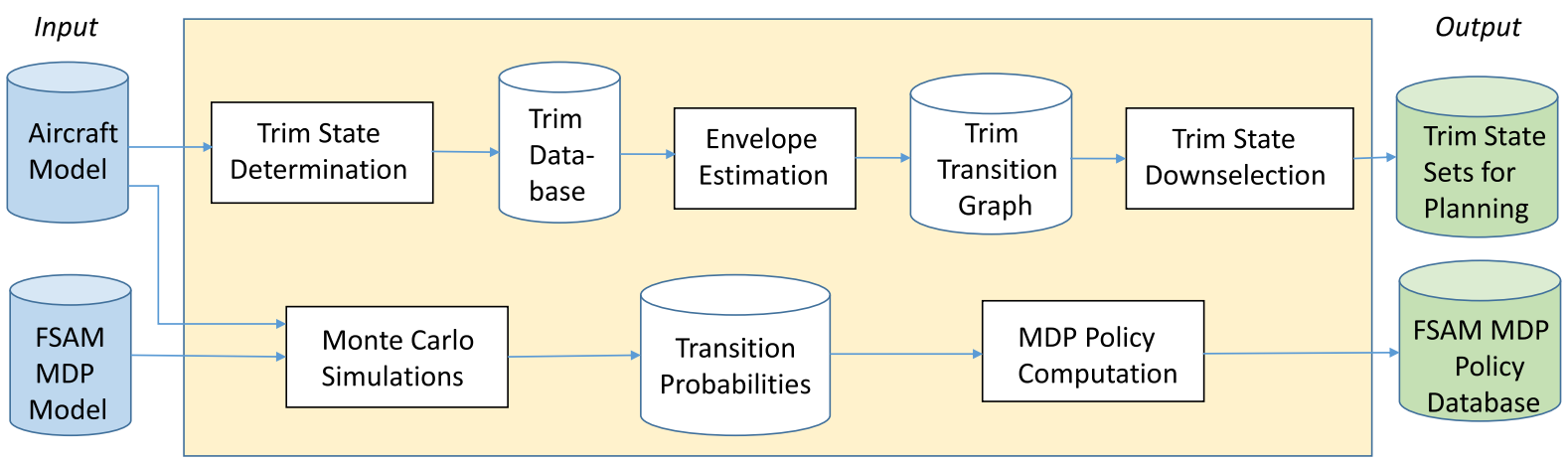

Fig. 4 EA-FMS offline processing for each nominal and exception database case.

Input

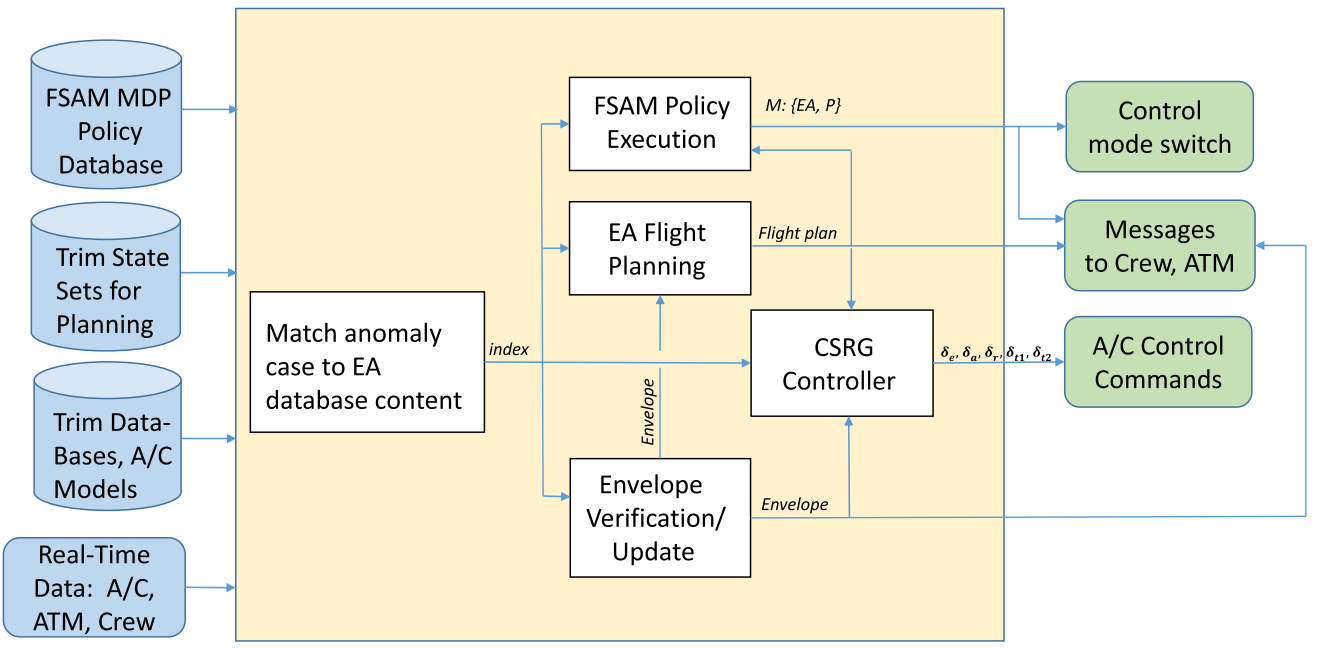

Fig. 5 EA-FMS online execution with exception case databases.

\section{B. Online Tasks}

Figure 5 shows the EA-FMS execution cycle when both nominal and exception (anomaly) cases are managed with database information generated in offline preprocessing. At the start of a flight, EAFMS runs the suite of FSAM, flight planning, and envelope verification (estimation) models/policies associated with the current flight phase. When an exception case is identified, it is matched or indexed to a set of EA databases, providing the appropriate MDP policy and trim information to EA-FMS. For rudder jam in flight, FSAM will execute the policy shown in Table 1. In parallel, the EA flight planner will generate a feasible flight plan for the given exception, such as an emergency landing plan for rudder jam. Realtime data is used by all EA-FMS modules, for example, to update physical state for all modules as well as pilot-specified mode $\bar{S}$ for FSAM

While $\bar{M}=P$, FSAM continues to monitor real-time data. If FSAM detects the need for EA control (TOGL action indicated), FSAM switches to EA control. If a prepared EA flight plan is ready, CSRG will follow it. If no such plan is ready, EA-FMS will first bring the aircraft to a feasible trim state. This trim state is used as the initial state for the EA flight planner (the AFP). As described in Sec. IV.B, the AFP selects a nearby landing site. It then builds a new flight plan from the stored trim state sets associated with the given exception case using the algorithm presented in Sec. IV.C. Note that, for this example, envelope estimation is not executed online because the envelope was precomputed offline. Instead, envelope estimation can run in the background to verify the CSRG controller is able to follow the flight plan and to potentially update the envelope as needed. Note that envelope update or online envelope estimation is required for new cases and for identified cases in which EA control doesn't succeed. Envelope estimation would then require support from system identification and potentially an adaptive control algorithm, but such cases are beyond the scope of this paper, which focuses on control surface (jam) cases.
For cases in which an FSAM policy is not available to mitigate LOC risk, FSAM will simply remain passive, deferring to the flight crew. The crew can be alerted that FSAM will not activate EA-FMS automatically, but the crew would be able to manually engage envelope-aware control should they elect this option.

\section{Rudder-Jam Case Study Results}

A demonstration of the proposed EA-FMS was performed using a rudder-jam scenario in a Twin Otter aircraft. This work employs an available nonlinear model of the aircraft [54]. Rudder jam is simulated by fixing the rudder deflection to $8 \mathrm{deg}$ once the jam occurs.ti This work makes the following assumptions:

1. Only a rudder jam occurs. There are no secondary anomalies such as hydraulic line failures, structural damage, etc.

2. Sensors failures do not occur.

3. The rudder jam is correctly diagnosed, and the jam angle is accurately measured.

4. The postjam aircraft performance model is accurate.

5. No wind is present.

6. The EA-FMS plan can terminate upon final approach. Touchdown and ground roll-out are not considered in this work.

7. The flight crew will support LOC avoidance with assistance from FSAM and EA-FMS.

8. A flight plan with a constant approach airspeed is acceptable without consideration of flaps, slats, gear, etc.

\footnotetext{
${ }^{\dagger}$ Although a true rudder jam would typically stop the rudder at some current deflection angle, this work simulates the fault as a ramp input from a nominal cruise trim state. The difference is that, before the rudder ramp input, flight is correctly governed by the nominal autopilot, which wouldn't inject a large rudder deflection under normal conditions. A large external disturbance, manual pilot input, sensor glitch, or software error could each cause such an event, but the LOC simulators used in this paper do not carefully model disturbances, pilot, sensor health, or software performance.
} 


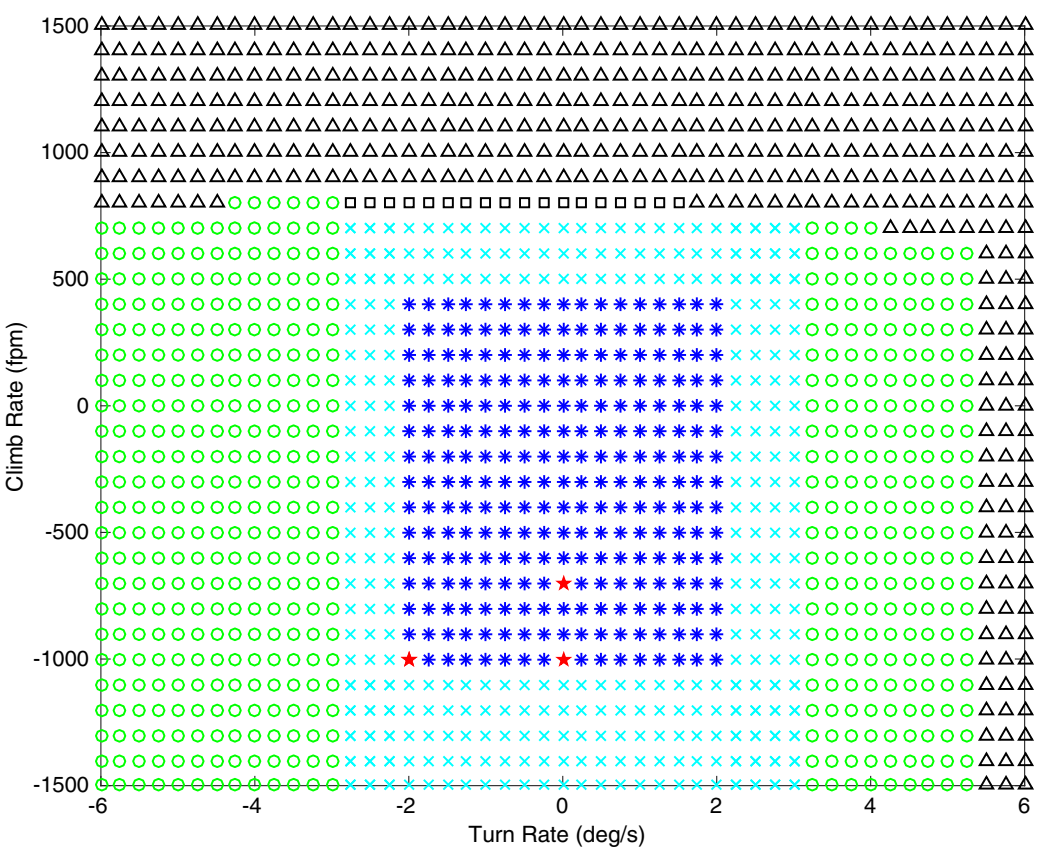

$\Delta$ Not a Trim Condition

口 Excessive Settling Time Trim Condition

- Unfeasible Transition Trim Condition

Feasible Transition Trim Condition

* Safe Feasible Transition Trim Condition

$\star$ Top Trim Conditions Selected for Flight Planning

Fig. 6 Trim states and transitions (airspeed $=130 \mathrm{KTAS}$; altitude $=8000 \mathrm{ft}$ ).

Future work required to relax these assumptions is discussed in the end of this section.

A twin-engine airplane subject to an asymmetric rudder-jam offset can be trimmed in different ways. First, differential thrust can allow trimming without sideslip. Another option is to use sideslip and aileron deflection to balance lateral moments. In our model, the high trim airspeeds do not allow differential thrust alone to trim the airplane with zero sideslip. For simplicity, thrust is therefore assumed
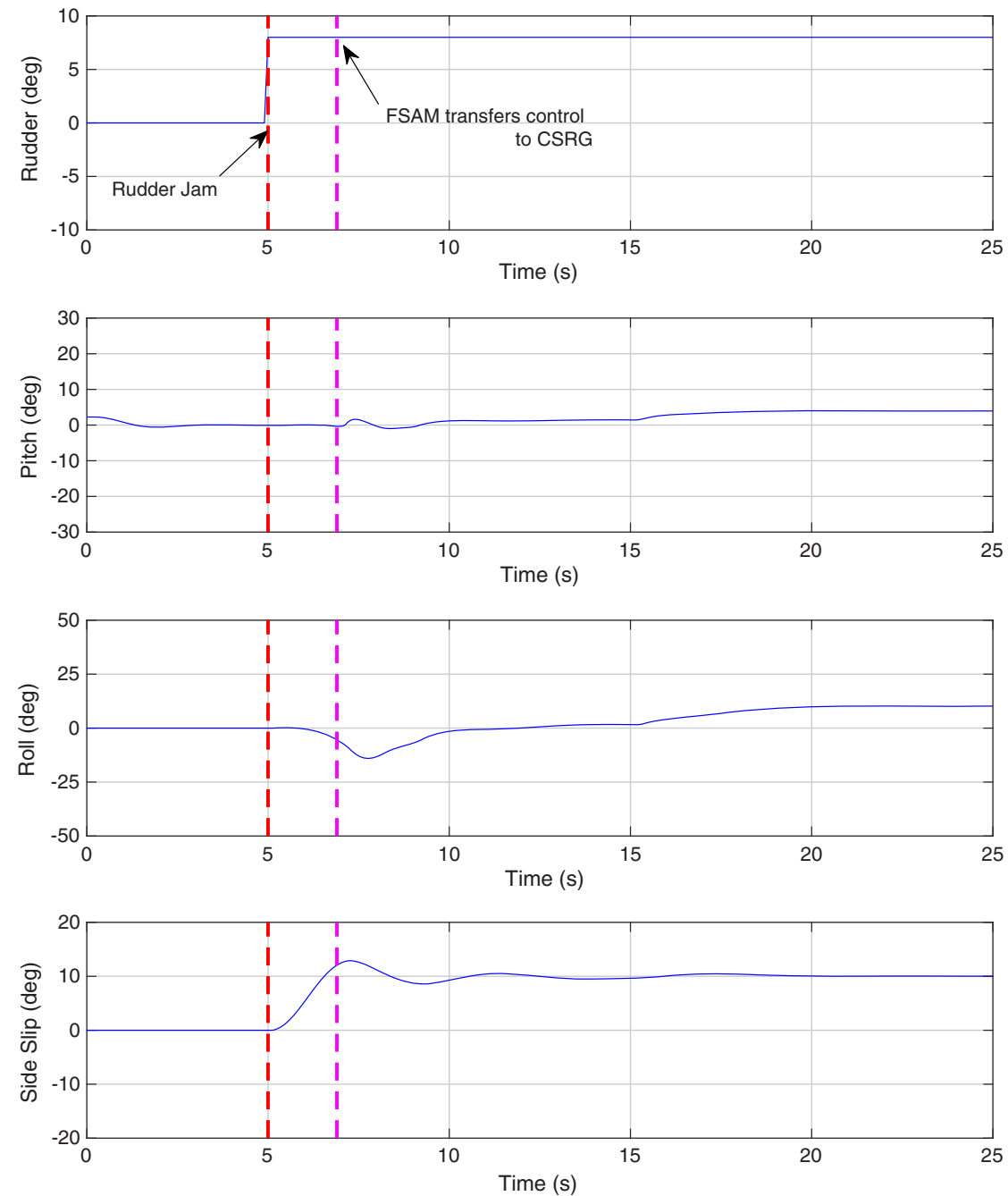

Fig. 7 Rudder-jam onset and transient response. 
symmetric throughout this paper because sideslip and aileron inputs can balance rudder-induced moments. An Linear Quadratic-Integral (LQ-I) control law [55] capable of maintaining a desired bank angle and flight path is used as the nominal autopilot and corresponds to $\bar{M}=P$. The CSRG described in Sec. III.B and Appendix A, is used as the envelope-aware safety controller $(M=\mathrm{EA})$.

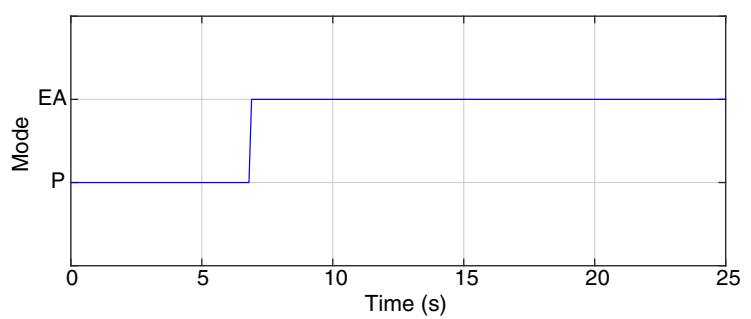

a)

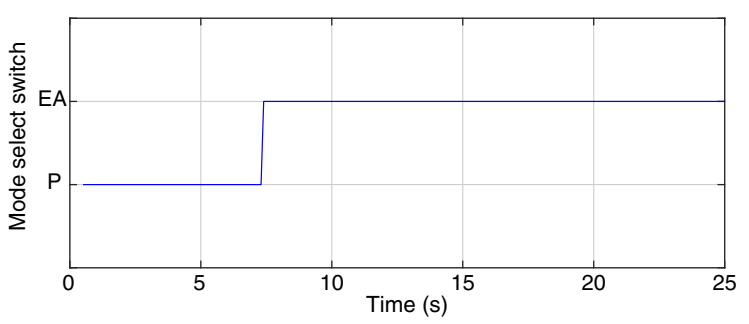

b)

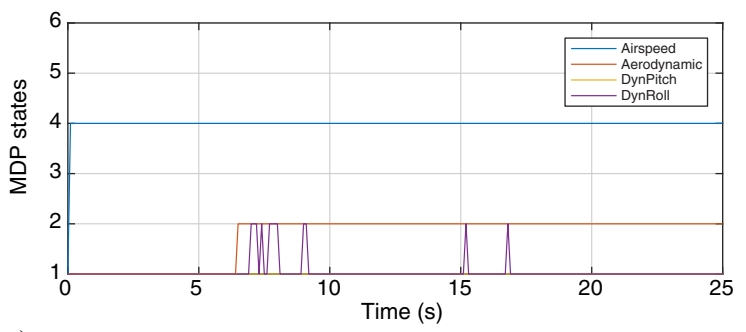

c)

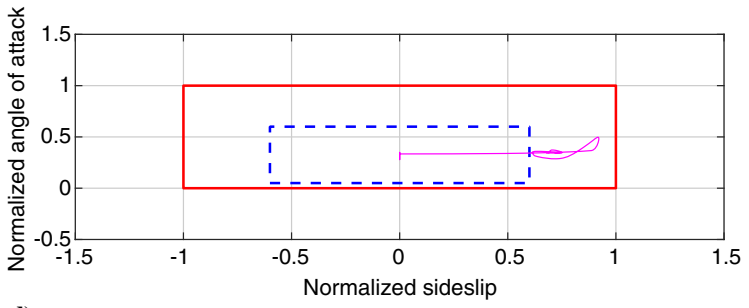

d)

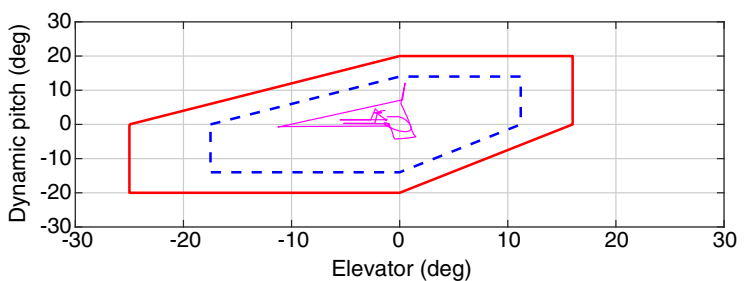

e)

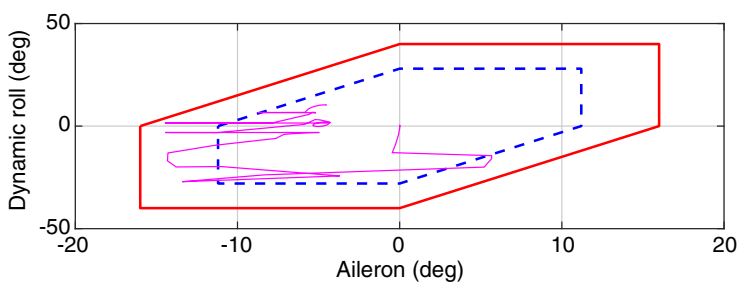

f)

Fig. 8 FSAM response.a) FSAM mode, b) FSAM mode select switch, c) MDP discrete states, d) Adverse aerodynamic envelope, e) Dynamic pitch control, and f) Dynamic roll control.
Figure $\underline{6}$ shows the trim conditions at $8000 \mathrm{ft}$ for an airspeed of 130 knots true airspeed (KTAS). The axis limits represent the range of climb and turn rates stored in the database. Each pair of turn-climb rates is classified in one of six groups.

1. Pairs that do not correspond to a trim condition.

2. Pairs that correspond to a trim condition, but whose closed loop dynamics require substantial settling time. Settling time can jeopardize the effectiveness of trim sequence flight planning due to extended transitions between trim states. Consequently, these states are not considered for flight planning.

3. Pairs that correspond to trim conditions whose transition to or from a wings-level constant altitude, or cruise, is not feasible. They are labeled Unfeasible Transition Trim Conditions.

4. Pairs that correspond to trim conditions whose transitions to and from a cruise condition using CSRG is feasible without violating any constraints. These pairs are named Feasible Transition Trim Conditions.

5. Pairs that correspond to Feasible Transition Trim Conditions which also maintain a sufficient margin away from unfeasible transition trim conditions. These pairs are named Safe Feasible Transition Trim Conditions.

6. Pairs that correspond to Safe Feasible Transition Trim Conditions ranked first to be used in path planning, as explained in Sec. IV.A.

Figure 6 represents the feasible transition trim points created for a single altitude. Such trim sets are examined for the initial altitude, a lower altitude close to the expected landing runways altitudes, and selected intermediate altitudes. The feasible trim point set list is constrained to those available at all examined altitudes. Because decreased air density (increased altitude) usually reduces the number of feasible transition trim points, trim states for the highest case study altitude are presented.

The case study simulation starts with the Twin Otter cruising at $8000 \mathrm{ft}$ three miles north-west of San Francisco International Airport (KSFO). The nominal FMS cruise autopilot is engaged. Figure 7 illustrates the onset of the rudder jam. The rudder jam is initiated $5 \mathrm{~s}$ into the simulation.
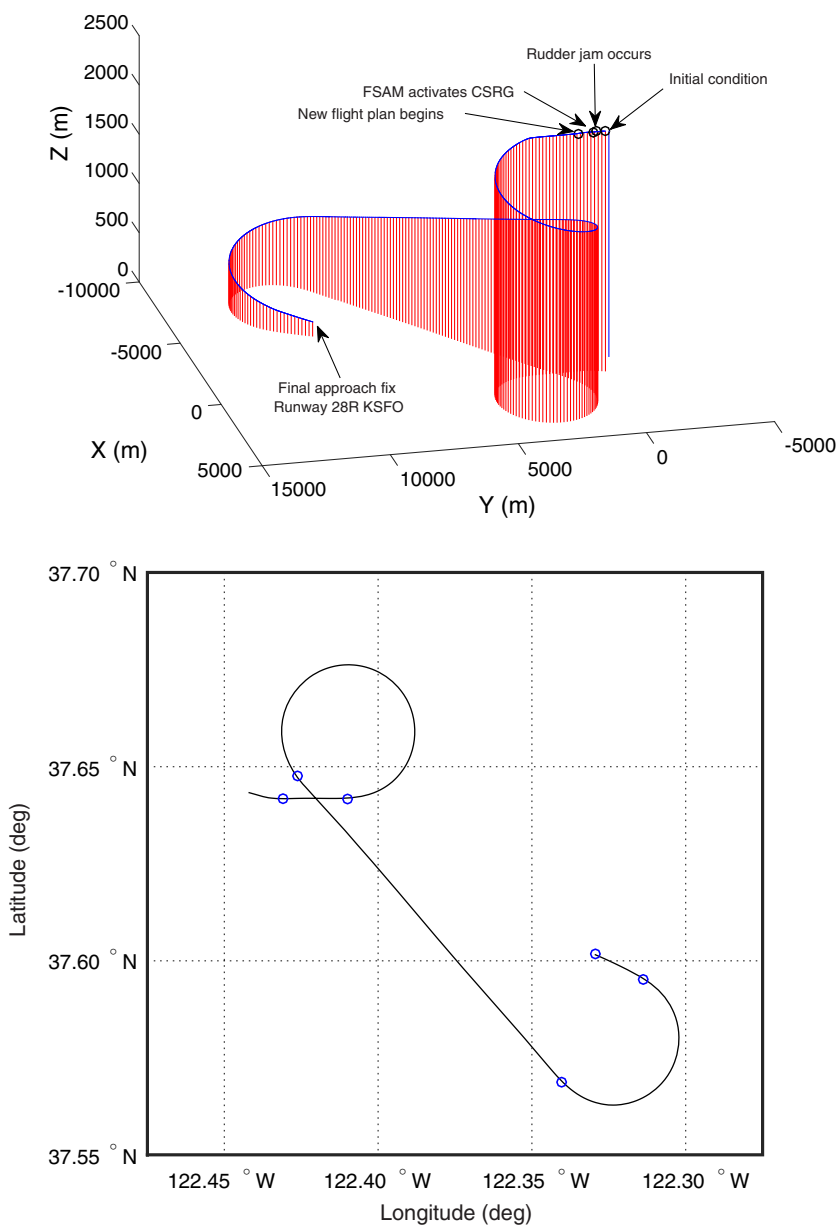

Fig. 9 Postfailure flight trajectory from 3-D (top) and 2-D overhead (bottom) viewpoints. 
Consequently, the sideslip angle starts to increase despite the usage of ailerons to counteract adverse roll due to the jammed rudder. Because the sideslip angle violates the inner thresholds of the adverse aerodynamic envelope (see Fig. 8), FSAM activates envelope-aware control, which prevents further envelope violations. Note that the simulation assumes the CSRG (envelope-aware control) is aware of the rudder failure, and so it uses the appropriate rudder-jam database control law. In this case study, control is never transferred back to the pilot per the FSAM model discussed previously, which allows the EA controller to fully execute its emergency landing plan, given concurrence from the crew via the $\bar{S}$ mode switch (see Fig. 8b).

When CSRG is initially activated by FSAM, it tries to stabilize the airplane to a suitable trim condition whose safe sets contain the aircraft state at the point of initiation. This ensures that the envelope constraints are never violated. The EA flight planner (AFP) builds a list of suitable runways, ranks them, and constructs a landing plan for the top-ranked runway. In this case study, the AFP selects KSFO 28R ranked first (see Appendix B). Guidance and CSRG track the given path to the runway. The top-ranked trim state set chosen by the flight planner is also presented in Fig. 6. Figure 9 presents the executed flight plan. This example highlights a tradeoff between using the "preferred" database trim state sequence and landing at the top-ranked runway. A robust AFP would explore multiple trim state sequences and multiple reachable runways to maximize the probability of finding at least one safe landing plan. If multiple safe landing plans are identified, AFP would either interact with the crew or internally trade off the top runway and preferred trim state "desirability." Maximizing options requires specification of a nontrivial set of trim state sequences in each anomaly database entry, in particular, at least a sufficient sequence set to support both left and right turns for each Dubins turn segment.

Figure 10 shows output from the guidance system after CSRG engagement. Note that additional trim states are used for accurate path tracking, motivating the use of a trim state set with appreciable margin from envelope boundaries as shown in Fig. 6. Figures 11-13 illustrate that the aircraft response with the CSRG satisfies imposed safety state and control constraints.

Although the example presented above illustrates EA-FMS application, it relies on a number of stated assumptions in future work. Winds of low intensity can be overcome by the controller or guidance system, but stronger steady winds must be incorporated into flight planning. Strong winds can also make final runway alignment challenging. The current landing site selection module mitigates such hazards by selecting runways with smaller crosswind components. Ground roll-out may also be challenging with rudder jam and low power conditions. Differential wheel braking and aerodynamic surface braking can be used in this case,
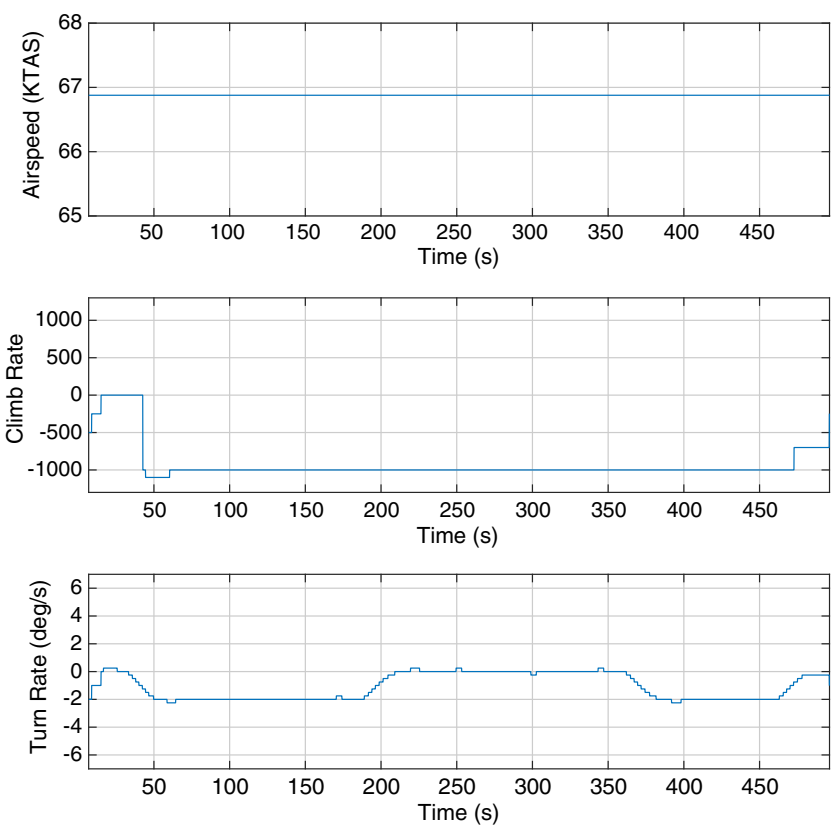

Fig. 10 Commanded trim conditions after CSRG engagement.
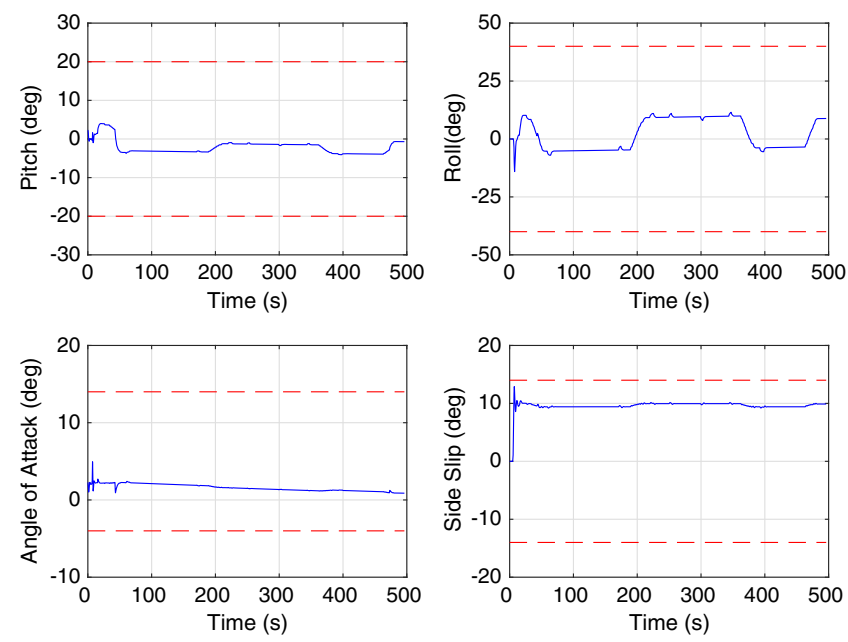

Fig. 11 Aircraft states when following new flight plan in EA mode.
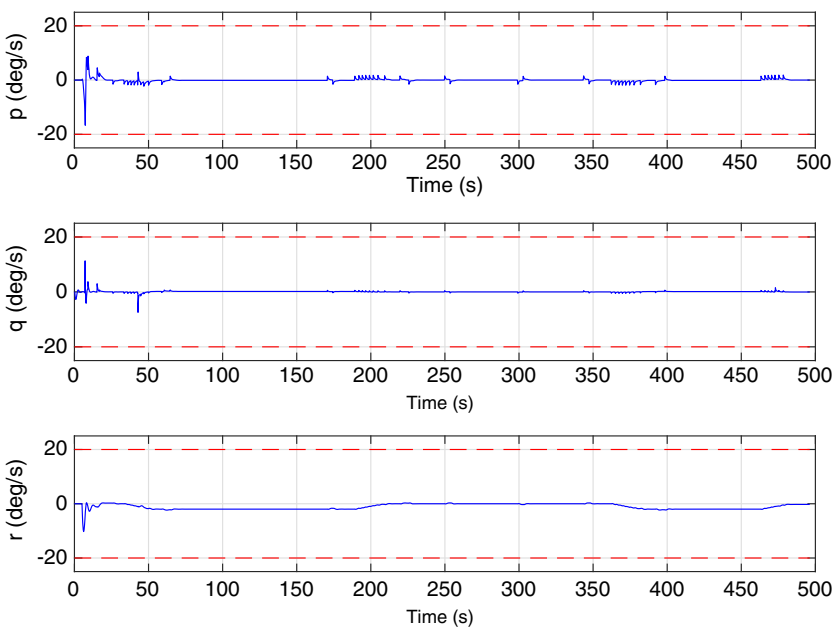

Fig. 12 Angular rates when following new flight plan in EA mode.
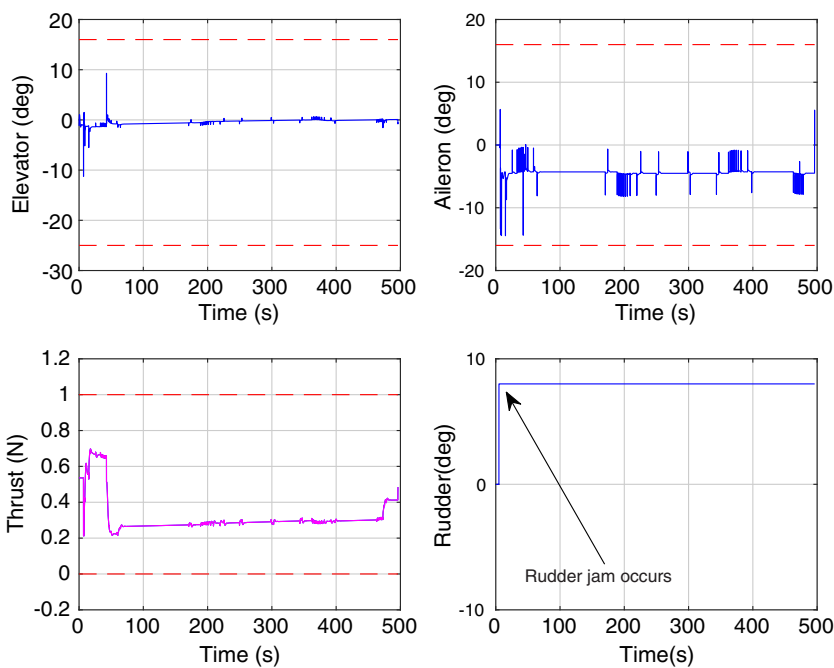

Fig. 13 Control inputs when following new flight plan in EA mode.

but specifics are not addressed in this paper. Sensor failures would also require additional consideration, although triply redundant architectures typically handle single failures internally.

\section{Conclusions}

This paper introduced the Envelope-Aware Flight Management System (EA-FMS) and presented its envelope estimation, adaptive 
(or envelope-aware) flight planning, and flight safety assessment and management (FSAM) functions. These modules were integrated to show EA-FMS loss of control (LOC) prevention, given a rudder actuator jam. A database of feasible trim states, transitions between these states, and control state reference governors (CSRG) can be constructed via envelope estimation over the suite of nominal and anomaly cases that can be modeled offline. This EA-FMS database can be queried online to provide "trusted" case-appropriate data for authority management (FSAM), flight planning, and CSRG control. The rudder-jam case study results illustrated database content and demonstrated that FSAM will override just in time to allow EA-FMS

The controller has the form $u=-K_{1} \Delta x-K_{2} x_{I}$. The values of $Q$ and $R$ matrices, as well as $K_{1}$ and $K_{2}$, for the trim condition presented above are

$$
\begin{gathered}
Q_{i j}=\left\{\begin{array}{cc}
\left(10 x_{i, \mathrm{lim}}{ }^{2}\right)^{-1} & \text { if }: i=j \\
0 & \text { otherwise }
\end{array}\right. \\
R_{i j}=10^{-3}\left\{\begin{array}{cc}
\left(u_{i, \mathrm{lim}}{ }^{2}\right)^{-1} & \text { if }: i=j \\
0 & \text { otherwise }
\end{array}\right.
\end{gathered}
$$

$$
K_{1}=\left[\begin{array}{cccccccc}
-0.6879 & 0.9765 & 0.0098 & 3.9230 & -0.3466 & -0.1784 & 0.0004 & -0.2344 \\
-0.6834 & 0.1502 & -0.8134 & 1.9774 & 3.4742 & 0.1984 & -0.0141 & 0.8499 \\
-0.1376 & -0.0101 & 0.0021 & -0.1940 & 1.7343 & 8.3152 & 2.2127 & 0.6346
\end{array}\right]
$$

to recover and safely land the disabled aircraft. Use of a "trusted database" approach to EA-FMS facilitates verification and validation to assure a safe landing despite high initial LOC risk.

\section{Appendix A: Linearized Aircraft Dynamics and Control Law Design for Envelope-Aware Control Mode}

A Twin Otter aircraft dynamics model is used in this work [54]. This work uses a power plant model from [54] modified to include altitude effects. The nonlinear aircraft model is linearized [56] about different trim conditions. The states for the linearized aircraft dynamics are $\Delta x=[\Delta \alpha, \Delta q, \Delta U, \Delta \theta, \Delta \beta, \Delta \phi, \Delta p, \Delta r]^{\top}$. Here, $\Delta$ indicates perturbations from trim conditions. Control inputs are elevator, total engine power (engines are assumed to give symmetric power), and aileron. The rudder is jammed and unavailable for control.

Consider a trim condition at an airspeed of $U_{0}=130$ KTAS, altitude of $h_{0}=8000 \mathrm{ft}$, flight path angle of $\gamma_{0}=0 \mathrm{deg}$, bank angle of $\phi_{0}=0 \mathrm{deg}$ with the rudder jammed at $\delta_{r 0}=8 \mathrm{deg}$. The corresponding control surface deflections and power required are $\delta_{a 0}=-4.509 \mathrm{deg}, \quad \delta_{e 0}=-1.365 \mathrm{deg}, \quad \delta_{t_{0}}=0.666 P_{\max (8000 \mathrm{ft})}$. Matrices $A$ and $B$ of the continuous time linearized dynamics for this trim conditions are given in Eqs. (A1) and (A2).

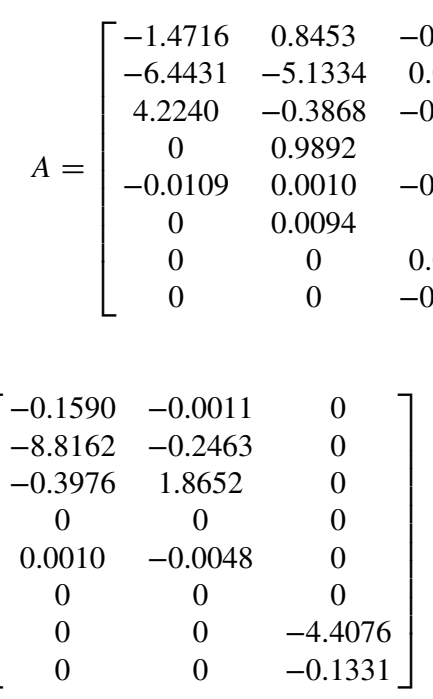

The control scheme is designed with LQ-I techniques. The state vector is extended with integrator states using the following $C_{I}$ matrix and a time step for discretization equal to $0.1 \mathrm{~s}$ :

$$
C_{I}=\left[\begin{array}{cccccccc}
-1 & 0 & 0 & 1 & 0 & 0 & 0 & 0 \\
0 & 0 & 1 & 0 & 0 & 0 & 0 & 0 \\
0 & 0 & 0 & 0 & 0 & 1 & 0 & 0
\end{array}\right]
$$

$$
K_{2}=\left[\begin{array}{ccc}
1.4892 & -0.0001 & 0.1314 \\
-1.2738 & -0.4429 & 0.5001 \\
-0.0221 & 0.0026 & 9.6308
\end{array}\right]
$$

The reference command is included using the following matrices:

$$
\bar{B}=\left[\begin{array}{c}
0_{8,3} \\
-I_{3 \times 3}
\end{array}\right] \quad \bar{D}=0_{11,3} \quad \Gamma=10^{5} I_{3 \times 3}
$$

The linearized model is constrained with respect to safety, control, and model validity. Safety constraints keep the aircraft within the flight envelope. Control constraints reflect ranges in which actuator positions can physically vary. Model validity constraints ensure the linearized model is sufficiently accurate and apply to both state variables and control inputs. The most limiting of the constraints is used for $Q$ and $R$ matrices. Safety and model validity constraints (with respect to state variables) are listed in Table A1.

Control and model validity (with respect to control inputs) constraints are listed in Table $\underline{\mathrm{A} 2}$

$\left.\begin{array}{cccc}-0.0000 & -0.0218 & -0.1754 & -0.0068 \\ 0 & 0 & 0 & 0 \\ -1.7877 & 1.6191 & -0.0883 & 0.1765 \\ 0 & 0 & 0 & -0.1467 \\ -0.1501 & 0.1407 & 0.0306 & -0.9701 \\ 0 & 0 & 1.0000 & 0.0632 \\ -2.2723 & 0 & -2.1828 & 0.2403 \\ 1.5372 & 0 & -0.2000 & -0.4196\end{array}\right]$

This work assumes the maximum power available at sea level for each engine is 579 equivalent shaft horsepower.

\section{Appendix B: Landing Site Search Parameters and Results}

The LSS runway ranking utility function in Eq. (7) contains a set of relative weighting terms. For this paper, weights $\left\{C_{1}^{-}, C_{2}, \ldots, C_{10}\right\}=$ $\{0.15,0.15,0.10,0.15,0,0,0.10,0.15,0.10,0.10\}$ were chosen. These rewards balance the terms but would require further input from the pilot community to be considered "optimal" with respect to user preferences. Term $q_{i}$ in Eq. (7) rewards the presence of an instrument approach, with the highest-precision instrument landing system and microwave landing system approaches receiving value 1.0 and others (including simplified directional facility, localizer, localizer-type directional aid, and interim standard microwave landing system) receiving value 0.5 . Hard runway surfaces (concrete or asphalt) are preferred with surface utility $q_{s}=1.0$, treated or gravel surfaces 
Table A1 State constraints

\begin{tabular}{lcc}
\hline \hline Variable & \multicolumn{1}{c}{ Safety constraint } & Model validity constraint \\
\hline Airspeed & $70 \leq \Delta U+U_{0} \leq 160 \mathrm{KTAS}$ & $-10 \leq \Delta U \leq 10 \mathrm{KTAS}$ \\
Angle of attack & $-4 \mathrm{deg} \leq \Delta \alpha+\alpha_{0} \leq 14 \mathrm{deg}$ & $-10 \mathrm{deg} \leq \Delta \alpha \leq 10 \mathrm{deg}$ \\
Pitch rate & $-20 \mathrm{deg} / \mathrm{s} \leq \Delta q+q_{0} \leq 20 \mathrm{deg} / \mathrm{s}$ & $-20 \mathrm{deg} / \mathrm{s} \leq \Delta q \leq 20 \mathrm{deg} / \mathrm{s}$ \\
Pitch angle & $-20 \mathrm{deg} \leq \Delta \theta+\theta_{0} \leq 20 \mathrm{deg}$ & $-20 \mathrm{deg} \leq \Delta \theta \leq 20 \mathrm{deg}$ \\
Sideslip & $-14 \mathrm{deg} \leq \Delta \beta+\beta_{0} \leq 14 \mathrm{deg}$ & $-10 \mathrm{deg} \leq \Delta \beta \leq 10 \mathrm{deg}$ \\
Bank angle & $-40 \mathrm{deg} \leq \Delta \phi+\phi_{0} \leq 40 \mathrm{deg}$ & $-20 \mathrm{deg} \leq \Delta \phi \leq 20 \mathrm{deg}$ \\
Roll rate & $-20 \mathrm{deg} / \mathrm{s} \leq \Delta p+r_{0} \leq 20 \mathrm{deg} / \mathrm{s}$ & $-20 \mathrm{deg} / \mathrm{s} \leq \Delta p \leq 20 \mathrm{deg} / \mathrm{s}$ \\
Yaw rate & $-20 \mathrm{deg} / \mathrm{s} \leq \Delta r+r_{0} \leq 20 \mathrm{deg} / \mathrm{s}$ & $-20 \mathrm{deg} / \mathrm{s} \leq \Delta r \leq 20 \mathrm{deg} / \mathrm{s}$ \\
\hline \hline
\end{tabular}

Table A2 Control constraints

\begin{tabular}{lcc}
\hline \hline Input & Control constraint & Model validity constraint \\
\hline Elevator & $-25 \mathrm{deg} \leq \Delta \delta_{e}+\delta_{e 0} \leq 16 \mathrm{deg}$ & $-10 \mathrm{deg} \leq \Delta \delta_{e} \leq 10 \mathrm{deg}$ \\
Power & $0 \leq \Delta \delta_{P}+\delta_{P 0} \leq 1$ & $-0.25 \leq \Delta \delta_{P} \leq 0.25$ \\
Aileron & $-16 \mathrm{deg} \leq \Delta \delta_{a}+\delta_{a 0} \leq 16 \mathrm{deg} / \mathrm{s}$ & $-10 \mathrm{deg} \leq \Delta \delta_{a} \leq 10 \mathrm{deg} / \mathrm{s}$ \\
\hline \hline
\end{tabular}

Table B1 LSS sorted runways results for the rudder-jam case study

\begin{tabular}{lcccccc}
\hline \hline Airport & Runway & Latitude, deg & Longitude, deg & Altitude, ft & Heading, deg & Utility \\
\hline KSFO & 28R & 37.613534 & -122.357141 & 13 & 298 & 0.946 \\
KSFO & 28L & 37.611712 & -122.358349 & 13 & 298 & 0.940 \\
KSFO & 19L & 37.627342 & -122.367111 & 10 & 208 & 0.912 \\
KSFO & 10L & 37.628739 & -122.393392 & 6 & 118 & 0.863 \\
KSFO & 10R & 37.626291 & -122.393105 & 7 & 118 & 0.856 \\
KOAK & 12 & 37.720062 & -122.242114 & 8 & 129 & 0.841 \\
\hline \hline
\end{tabular}

each receive $q_{s}=0.5$, and turf or dirt each receive $q_{s}=0.25$. The presence of a manned air traffic control tower is also rewarded by $q_{\text {atc }}=1.0$. Presence of emergency responders $q_{\mathrm{ff}}$ is rewarded based on Federal Aviation Regulations (FAR) 139.315, with indices A receiving $q_{\mathrm{ff}}=0.5$, B receiving $q_{\mathrm{ff}}=0.8$, and $\mathrm{C}$, D, or E each receiving $q_{\mathrm{ff}}=1.0$. Available resources for the next flight $q_{\mathrm{nf}}$ sum to an overall maximum total of $1.0 ; q_{\mathrm{nf}}$ is composed of fuel (value $0.25)$, airframe maintenance ( 0.25 for major, 0.125 for minor), powerplant maintenance ( 0.25 for major, 0.125 for minor), and bottled oxygen ( 0.25 for high and low pressure, 0.125 for high only, 0.125 for low only). Wind terms associated with $C_{5}$ and $C_{6}$ are assigned zero weights in this paper because wind was not modeled. Table B1 presets the top seven runways selected by LSS in the case study.

\section{Acknowledgments}

This work was supported in part by the National Aeronautics and Space Administration under Cooperative Agreement NNX12AM54A. The first author is supported in part by CAPES-Brazil through the Science Without Borders program, process number BEX 19130/12-0. This work does not reflect official positions of ANAC-Brazil. The authors thank University of Michigan student Chaaru Raghavan for contributions to the EA-FMS software.

\section{References}

[1] Belcastro, C. M., and Jacobson, S. R., "Future Integrated System Concepts for Preventing Aircraft Loss-of-Control Accidents," Proceedings of AIAA Guidance Navigation, and Control Conference, AIAA Paper 2010-8142, 2010. doi: $10.2514 / 6.2010-8142$

[2] Traverse, P., "Airbus Electrical Flight Controls: A Family of FaultTolerant Systems," Digital Avionics Handbook, 3rd ed., edited by Spitzer, C. R., Farrell, U., and Ferrell, T., CRC Press, Boca Raton, FL, 2015, pp. 31-1-31-18, Chap. 31

[3] Balachandran, S., and Atkins, E. M., "Flight Safety Assessment and Management During Takeoff," AIAA Infotech@Aerospace Conference, AIAA Paper 2013-4805, 2013. doi: $10.2514 / 6.2013-4805$
[4] Balachandran, S., and Atkins, E. M., "An Evaluation of Flight Safety Assessment and Management to Avoid Loss of Control During Takeoff," AIAA Guidance, Navigation and Control Conference, AIAA Paper 2014-0785, 2014. doi:10.2514/6.2014-0785

[5] Balachandran, S., and Atkins, E. M., "A Constrained Markov Decision Process Framework for Flight Safety Assessment and Management," AIAAInfotech@Aerospace Conference, AIAA Paper 2015-0115, 2015. doi:10.2514/6.2015-0115

[6] Balachandran, S., and Atkins, E. M., "Flight Safety Assessment and Management for Takeoff Using Deterministic Moore Machines," Journal of Aerospace Information Systems, Vol. 12, No. 9, 2015, pp. 599-615. doi:10.2514/1.I010350

[7] Balachandran, S., and Atkins, E. M., "A Markov Decision Process Framework for Flight Safety Assessment and Management," Journal of Guidance, Control, and Dynamics (accepted for publication).

[8] McDonough, K., Kolmanovsky, I., and Atkins, E. M., "Recoverable Sets of Initial Conditions and Their Use for Aircraft Flight Planning After a Loss of Control Event," Proceedings of AIAA Guidance Navigation, and Control Conference, AIAA Paper 20140786, 2014. doi: $10.2514 / 6.2014-0786$

[9] McDonough, K., and Kolmanovsky, I., "Integrator Resetting for Enforcing Constraints in Aircraft Flight Control Systems," Proceedings of AIAA Guidance Navigation, and Control Conference, AIAA Paper 2015-1995, 2015.

doi:10.2514/6.2015-1995

[10] Di Donato, P. F. A., and Atkins, E. M., "An Off-Runway Emergency Landing Aid for a Small Aircraft Experiencing Loss of Thrust," AIAA Infotech@Aerospace Conference, AIAA Paper 2015-1798, 2015. doi:10.2514/6.2015-1798

[11] Yu, M.-J., McDonough, K., Bernstein, D. S., and Kolmanovsky, I., "Retrospective Cost Model Refinement for Aircraft Fault Signature Detection," 2014 American Control Conference (ACC), IEEE Publ., Piscataway, NJ, 2014, pp. 2486-2491. doi:10.1109/ACC.2014.6858876

[12] Yu, M. J., Zhong, J., Atkins, E. M., Kolmanovsky, I., and Bernstein, D. S., "Trim-Commanded Adaptive Control for Waypoint-Defined Trajectory Following," Proceedings of AIAA Guidance Navigation, and Control Conference, AIAA Paper 2013-5000, 2013. doi: $10.2514 / 6.2013-5000$

[13] Yi, G., Zhong, J., Atkins, E. M., and Wang, C., "Trim State Discovery with Physical Constraints," Journal of Aircraft, Vol. 52, No. 1, 2014, 
pp. 1-17.

doi: $10.2514 / 1 . C 032619$

[14] Atkins, E. M., Portillo, I. A., and Strube, M. J., "Emergency Flight Planning Applied to Total Loss of Thrust," Journal of Aircraft, Vol. 43, No. 4, 2006, pp. 1205-1216.

doi: $10.2514 / 1.18816$

-[15] Borst, C., Grootendorst, F. H., Brouwer, D. I. K., Bedoya, C., Mulder, M., and van Paassen, M. M., "Design and Evaluation of a Safety Augmentation System for Aircraft," Journal of Aircraft, Vol. 51, No. 1, 2013, pp. 12-22. doi:10.2514/1.C031500

[16] Gingras, D. R., Barnhart, B., Ranaudo, R., Ratvasky, T. P., and Morelli, E., "Envelope Protection for In-Flight Ice Contamination," 47th AIAA Aerospace Sciences Meeting, AIAA Paper 2009-1458, 2009, pp. 20091458.

doi: $10.2514 / 6.2009-1458$

[17] Gregory, I. M., Cao, C., Xargay, E., Hovakimyan, N., and Zou, X., "L1 Adaptive Control Design for NASA AirSTAR Flight Test Vehicle," Proceedings of AIAA Guidance, Navigation, and Control Conference, Vol. 5738, AIAA Paper :2009-5738, 2009. doi: $10.2514 / 6.2009-5738$

[18] Chongvisal, J., Tekles, N., Xargay, E., Talleur, D., Kirlik, A., and Hovakimyan, N., "Loss-of-Control Prediction and Prevention for NASA's Transport Class Model," Proceedings of AIAA Guidance, Navigation, and Control Conference, AIAA Paper 2014-0784, 2014. doi: $10.2514 / 6.2014-0784$

[19] Tekles, E. X., Choe, R., Hovakimyan, N., Gregory, I., and Holzapfel, F., "Flight Envelope Protection for NASA's Transport Class Model," Proceedings of AIAA Guidance, Navigation, and Control Conference, AIAA Paper 2014-0269, 2014. doi:10.2514/6.2014-0269

[20] Chowdhary, G., Johnson, E. N., Chandramohan, R., Kimbrell, M. S., and Calise, A., "Guidance and Control of Airplanes Under Actuator Failures and Severe Structural Damage," Journal of Guidance, Control, and Dynamics, Vol. 36, No. 4, 2013, pp. 1093-1104.

[21] Wilborn, J. E., and Foster, J. V., "Defining Commercial Transport Loss-of-Control: A Quantitative Approach," Proceedings of AIAA Atmospheric Flight Mechanics Conference and Exhibit, AIAA Paper 2004-4811, 2004.

doi:10.2514/6.2004-4811

[22] Kwatny, H. G., Dongmo, J.-E. T., Chang, B.-C., Bajpai, G., Yasar, M., and Belcastro, C., "Aircraft Accident Prevention: Loss-of-Control Analysis," Proceedings of AIAA Guidance, Navigation and Control Conference, AIAA Paper 2009-6256, 2009. doi:10.2514/6.2009-6256

[23] Kwatny, G., and Allen, R. C., "Safe Set Maneuverability of Impaired Aircraft," Proceedings of AIAA Guidance Navigation and Control Conference, AIAA Paper 2012-4405, 2012, pp. 12-16. doi: $10.2514 / 6.2012-4405$

[24] Lygeros, J., "On Reachability and Minimum Cost Optimal Control," Automatica, Vol. 40, No. 6, 2004, pp. 917-927. doi:10.1016/j.automatica.2004.01.012

[25] Schuet, S., Lombaerts, T., Acosta, D., Wheeler, K., and Kaneshige, J., "An Adaptive Nonlinear Aircraft Maneuvering Envelope Estimation Approach for Online Applications," Proceedings of AIAA Guidance, Navigation and Control Conference, AIAA Paper 2014-0268, Jan. 2014. doi: $10.2514 / 6.2014-0268$

[26] Bayen, A. M., Mitchell, I. M., Osihi, M. K., and Tomlin, C. J., "Aircraft Autolander Safety Analysis Through Optimal Control-Based Reach Set Computation," Journal of Guidance, Control, and Dynamics, Vol. 30, No. 1, 2007, pp. 68-77. doi: $10.2514 / 1.21562$

[27] Pandita, R., Chakraborty, A., Seiler, P., and Balas, G., "Reachability and Region of Attraction Analysis Applied to GTM Dynamic Flight Envelope Assessment," Proceedings of AIAA Guidance, Navigation, and Control Conference, AIAA Paper 2009-6258, 2009. doi: $10.2514 / 6.2009-6258$

[28] Tang, L., Roemer, M., Ge, J., Crassidis, A., Prasad, J., and Belcastro, C., "Methodologies for Adaptive Flight Envelope Estimation and Protection," Proceedings of AIAA Guidance, Navigation, and Control Conference, AIAA Paper 2009-6260, 2009. doi: $10.2514 / 6.2009-6260$

[29] Oort, E., Chu, Q., and Mulder, J., "Maneuver Envelope Determination Through Reachability Analysis," Advances in Aerospace Guidance and Control, edited by Holzapfel, F., and Theil, S., Springer, Berlin, 2011, pp. 91-102. doi:10.1007/978-3-642-19817-5_8

-[30] McDonough, K., and Kolmanovsky, I., "Controller State and Reference Governors for Discrete-Time Linear Systems with Pointwise-in-Time
State and Control Constraints," American Control Conference (ACC), IEEE Publ., Piscataway, NJ, 2015. doi:10.1109/ACC.2015.7171890

[31] McDonough, K., "Developments in Stochastic Fuel Efficient Cruise Control and Constrained Control with Applications to Aircraft," Ph.D. Thesis, The Univ. of Michigan, Ann Arbor, Michigan, 2015. doi:2027.42/111399

[32] Meuleau, N., Plaunt, C., Smith, D. E., and Smith, T. B., "An Emergency Landing Planner for Damaged Aircraft," Proceedings of the Twenty First Innovative Applications of Artificial Intelligence Conference, AAAI Press, Menlo Park, CA, 2009, pp. 114-121, http://aaai.org/ocs/ index.php/IAAI/IAAI09/paper/view/268.

[33] Dubins, L. E., "On Curves of Minimal Length with a Constraint on Average Curvature, and with Prescribed Initial and Terminal Positions and Tangents," American Journal of Mathematics, Vol. 79, No. 3, 1957, pp. 497-516. doi: $10.2307 / 2372560$

[34] Chitsaz, H., and LaValle, S. M., "Time-Optimal Paths for a Dubins Airplane," Decision and Control, 2007 46th IEEE Conference on IEEE, IEEE Publ., Piscataway, NJ, 2007, pp. 2379-2384. doi:10.1109/CDC.2007.4434966

[35] Hota, S., and Ghose, D., "Optimal Path Planning for an Aerial Vehicle in 3D Space," 49th IEEE Conference on Decision and Control (CDC), IEEE Publ., Piscataway, NJ, 2010, pp. 4902-4907. doi: $10.1109 / C D C .2010 .5717246$

[36] Owen, M., Beard, R. W., and McLain, T. W., "Implementing Dubins Airplane Paths on Fixed-Wing UAVs," Handbook of Unmanned Aerial Vehicles, Springer, Berlin, 2015, pp. 1677-1701. doi:10.1007/978-90-481-9707-1

[37] Tang, Y., Atkins, E., and Sanner, R., "Emergency Flight Planning for a Generalized Transport Aircraft with Left Wing Damage," Proceedings of AIAA Guidance, Navigation and Control Conference, AIAA Paper 2007-6873, 2007, pp. 20-23. doi: $10.2514 / 6.2007-6873$

[38] Frazzoli, E., "Maneuver-Based Motion Planning and Coordination for Single and Multiple UAVs," First Technical Conference and Workshop on Unmanned Aerospace Vehicles, AIAA Paper 2002-3472, 2002. doi: $10.2514 / 6.2002-3472$

[39] Delahaye, D., Puechmorel, S., Tsiotras, P., and Feron, E., "Mathematical Models for Aircraft Trajectory Design: A Survey," Air Traffic Management and Systems, Springer, Berlin, 2014, pp. 205-247. doi:10.1007/978-4-431-54475-3

[40] Jardin, M. R., and Bryson, A. E., "Methods for Computing MinimumTime Paths in Strong Winds," Journal of Guidance, Control, and Dynamics, Vol. 35, No. 1, 2012. pp. 165-171. doi: $10.2514 / 1.53614$

[41] Schouwenaars, T., How, J., and Feron, E., "Receding Horizon Path Planning with Implicit Safety Guarantees," American Control Conference, 2004. Proceedings of the 2004, IEEE Publ., Piscataway, NJ, 2004, pp. 5576-5581.

[42] Tomlin, C., Pappas, G. J., and Sastry, S., "Conflict Resolution for Air Traffic Management: A Study in Multiagent Hybrid Systems," Transactions on Automatic Control, Vol. 43, No. 4, 1998, pp. 509-521. doi:10.1109/9.664154

[43] Srivatsan, R., Downing, R. D., and Bryant, H. W., "Development of a Takeoff Performance Monitoring System," Journal of Guidance, Control, and Dynamics, Vol. 10, No. 5, 1987, pp. 433-440. doi: $10.2514 / 3.20237$

[44] Kochenderfer, M. J., and Chryssanthacopoulos, J. P., "A DecisionTheoretic Approach to Developing Robust Collision Avoidance Logic," Intelligent Transportation Systems (ITSC), 2010 13th International IEEE Conference on, IEEE, IEEE Publ., Piscataway, NJ, 2010. doi:10.1109/ITSC.2010.5625063

[45] Armand, J., Lignee, R., and Villaume, F., "The Runway Overrun Prevention System," Safety First-The Airbus Safety Magazine, Issue 8, Airbus S.A.S., July 2009.

[46] Burns, A., Harper, D., Barfield, A., Whitcomb, S., and Jurusik, B., "Auto GCAS for Analog Flight Control System," Digital Avionics Systems Conference (DASC), 2011 IEEE/AIAA 30th, IEEE Publ., Piscataway, NJ, 2011, pp. 8C5-1-8C5-11. doi:10.1109/DASC.2011.6096148

[47] Balachandran, S., and Atkins, E. M., "An Autonomous Override System to Prevent Airborne Loss of Control," Proceedings of the Thirtieth AAAI Conference on Artificial Intelligence and the Twenty-Eighth Conference on Innovation Applications of Artificial Intelligence, AAAI Press, Palo Alto, CA, 2016, pp. 3991-3396, http://www.aaai.org/ocs/ index.php/IAAI/IAAI16/paper/view/12161.

[48] Balachandran, S., Ozay, N., and Atkins, E. M., "Verification Guided Refinement of a Flight Safety Assessment and Management System 
for Takeoff," Journal of Aerospace Information Systems (accepted for publication).

[49] Kolmanovsky, I., and Gilbert, E. G., "Theory and Computation of Disturbance Invariant Sets for Discrete-Time Linear Systems," Mathematical Problems in Engineering, Vol. 4, No. 4, 1998, pp. 317-367. doi:10.1155/S1024123X98000866

[50] Weiss, A., Petersen, C., Baldwin, M., Erwin, R. S., and Kolmanovsky, I., "Safe Positively Invariant Sets for Spacecraft Obstacle Avoidance," Journal of Guidance, Control, and Dynamics, Vol. 38, No. 4, 2015, pp. 720-732. doi:10.2514/1.G000115

[51] Federal Aviation Administration, "Airport Data \& Contact Information," http://www.faa.gov/airports/airport_safety/airportdata_5010/ [accessed 17 Nov. 2015].
[52] Puterman, M. L., Markov Decision Process: Discrete Stochastic Dynamic Programming, John Wiley \& Sons, Hoboken, NJ, 1994, pp. 17-260.

[53] Balachandran, S., McDonough, K., di Donato, P., Atkins, E., and Kolmanovsky, I., "Envelope Aware Flight Management to Reduce Icing-Induced Loss of Control Risk," Journal of Guidance, Control, and Dynamics, 2015 (in preparation).

[54] Melody, J. W., "Inflight Characterization of Aircraft Icing," Ph.D. Thesis, Univ. of Illinois at Urbana-Champaign, 2004. doi:2142/80862

[55] Kirk, D. E., Optimal Control Theory: An Introduction, Prentice-Hall, Upper Saddle River, NJ, 1970, pp. 53-95, Chap. 3.

[56] Stevens, L. B., and Lewis, L. F., Aircraft Control and Simulation, Wiley, Hoboken, NJ, 2003, pp. 1-137, Chaps. 1-2. 
This article has been cited by:

1. Pedro F. A. Di Donato, Ella M. Atkins. Three-dimensional dubins path generation and following for a UAS glider 294-303. [Crossref]

2. Emanuele Garone, Stefano Di Cairano, Ilya Kolmanovsky. 2017. Reference and command governors for systems with constraints: A survey on theory and applications. Automatica 75, 306-328. [Crossref] 